\title{
Ethnomedicinal Plants of Hasankeyf (Batman-Turkey)
}

\author{
Yeter Yeşil ${ }^{1 *}$ and Ilyas Inal ${ }^{1}$ \\ ${ }^{1}$ Faculty of Pharmacy, Department of Pharmaceutical Botany, Istanbul University, Istanbul, Turkey
}

Hasankeyf is an ancient city, dating back to more than 10,000 years, in the Southeast Anatolia Region of Turkey. The area is separated by the Tigris River on both sides and located in the Batman province. However, as a result of a dam project, in February 2020, this ancient city and some of its surrounding villages were totally flooded. The residents were moved to new settlements. This study aimed to prevent the possible loss of ethnomedicinal knowledge of plants due to migration as well as to pass on this knowledge to the future generations. The field studies were conducted between March 2017 and November 2019 in the city center and 22 rural settlements of Hasankeyf. Also, the areas where intensive migration was experienced were visited frequently. Interviews were conducted with a total of 131 participants (76 women and 55 men) while gathering plants with them. Information was collected through interviews and questionnaires. The results were analyzed by quantitative indices of information consensus factor (FIC) and use value

OPEN ACCESS

Edited by: Andrea Pieroni,

University of Gastronomic Sciences, Italy

Reviewed by: Mark Nesbitt,

Royal Botanic Gardens, Kew, United Kingdom Fatih Satı,

Balıkesir University, Turkey

*Correspondence: Yeter Yeşil yesily@istanbul.edu.tr

Specialty section: This article was submitted to Ethnopharmacology,

a section of the journal

Frontiers in Pharmacology

Received: 01 November 2020 Accepted: 30 December 2020 Published: 11 March 2021

Citation: Yeşil Y and Inal I (2021) Ethnomedicinal Plants of Hasankeyf (Batman-Turkey). Front. Pharmacol. 11:624710. doi: 10.3389/fphar.2020.624710 (UV). A total of 94 plant taxa belonging to 40 families were identified in the study area. The most common medicinal plant families are Lamiaceae (13), Asteraceae (8), Rosaceae (6), Malvaceae (6), Amaryllidaceae (5), Brassicaceae (4), and Solanaceae (4). The most common preparations were infusion, fresh application, and crushing. The taxa having the highest count of use value (UV) were Teucrium polium, Matricaria aurea, Urtica dioica, Mentha Iongifolia, and Quercus brantii. Besides, the recorded ailments were grouped into categories based on information provided by the interviewees. The most important use categories among the informants were diabetes, gastrointestinal disorders, respiratory disorders, and dermatological disorders. The present study represents the first medical-ethnobotanical documentation and analysis of the traditional use of medicinal plants in Hasankeyf.

Keywords: medicinal plants, ethnobotany, ancient city, mesopotamia, anatolia

\section{INTRODUCTION}

Medicinal plants have been used globally throughout the centuries to treat various disorders and ailments. About $80 \%$ of the world population uses traditional medicine for primary health care needs (WHO, 1993). More than 50,000 of approximately 422,000 flowering plants in the world are used for medicinal purposes (Hamilton, 2004). The Covid 19 global epidemic has prompted renewed interest in medicinal plants (Vandebroek et al., 2020).

Turkey has a rich flora, with about 11.700 species (Davis, 1965-1985; Davis et al., 1988; Güner et al., 2000; Güner et al., 2012; Güner, 2014; Güner et al., 2018) and an endemism rate of 34\% (Güner, 2014). The majority of people who live in rural areas make use of this rich diversity of plants. Systematic ethnobotanical studies only began in the mid-1990s (Ertuğ and Güner, 2014). 


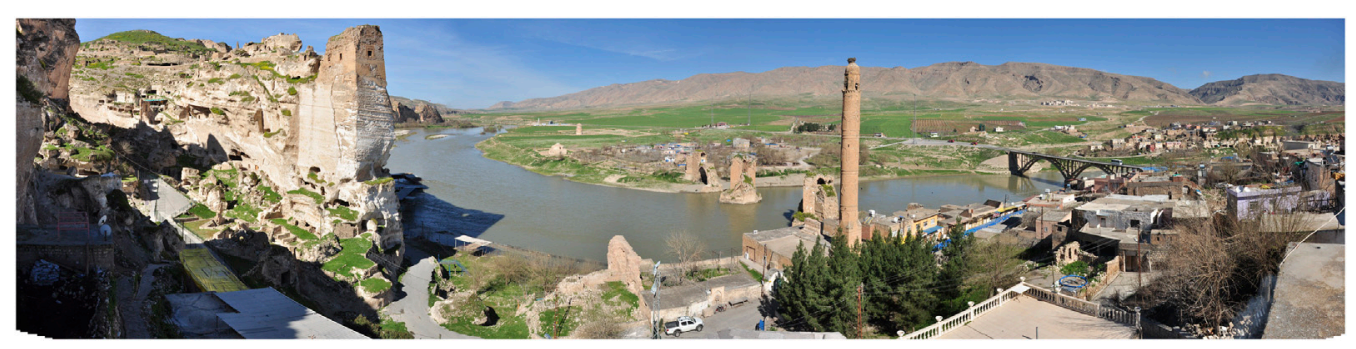

FIGURE 1 | Hasankeyf center with historical construction remains 2013

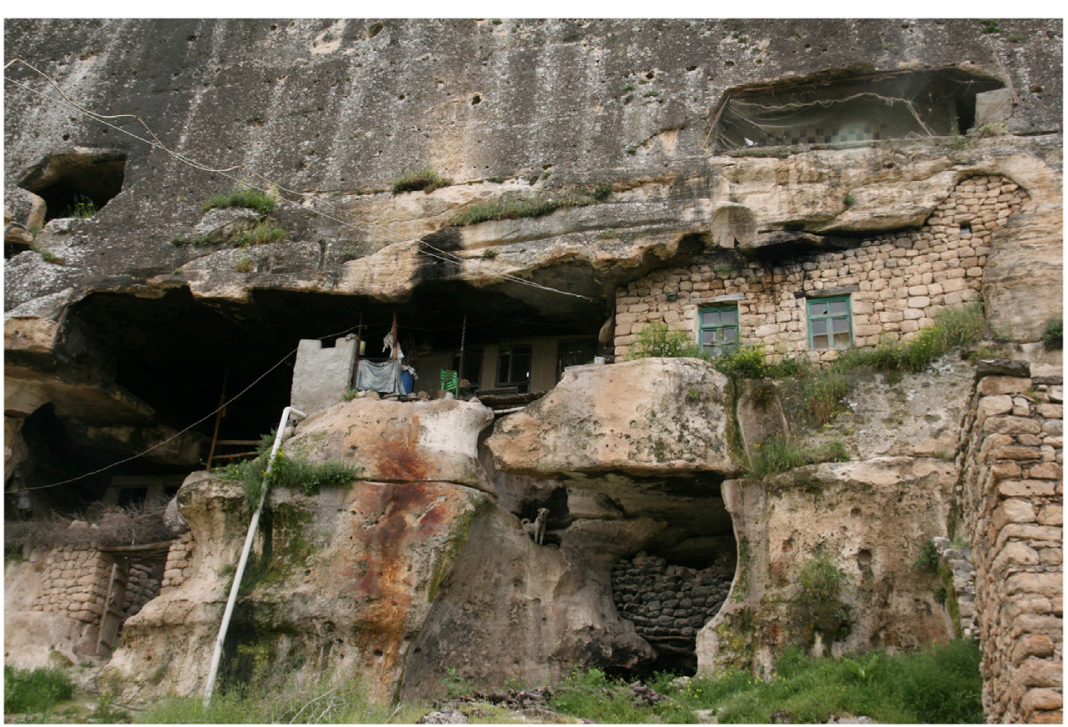

FIGURE 2 | A cave house in the ancient city, 2019.

According to the database of Turkish Folk Medicines Knowledge Base (TUHIB), the number of medicinal plant species in Turkey is more than 1,000 (Yeşilada, 2002; Yeşilada, 2005). In recent years, many studies have been published in Turkey (Günbatan et al., 2016; Korkmaz et al., 2016; Paksoy et al., 2016; Uzun and Kaya, 2016; Baykal and Atamov, 2017; Bulut et al., 2017; Güneş et al., 2017; Karc1 et al., 2017; Kartal and Güneş, 2017; Özdemir and Kültür, 2017; Akgül et al., 2018; Sargin and Büyükcengiz, 2018; Nacakç1 and Dutkuner, 2018; Gürbüz et al., 2019; Karakaya et al., 2019; Nadiroğlu et al., 2019; Polat, 2019; Çelik and Yeşil, 2020; Güler et al., 2020; Kaya et al., 2020; Kılıç et al., 2020). However, in southeastern Turkey, which covers the study area, only a limited number of ethnobotanical studies have been conducted (Gençay, 2007; Akan et al., 2013; Akgül et al., 2018; Bulut et al., 2019; Yeşil et al., 2019; Yeşil and Inal, 2019; Kılıç et al., 2020).

Hasankeyf is an ancient settlement located in the southeast of Turkey (Batman province) (Figure 1). It is located on the Tigris River in the valley extending from the Persian Gulf, the place of a medieval settlement. Dating back to the Middle Bronze Age, the settlement later became a center on the Silk Road. Life in the region was influenced by various powers from the prehistoric times to the Romans and Selcuks (Ahunday and Balk1z, 2009). The name "Hasankeyf" is derived from "kepa, kipas, kefa, kaife," which means "rock" in Arami or Arameik, Assyrian, Hebrew, Syriac, and Arabic language. The Assyrians named the settlement as "Castrum Kepha" (Rock Castle) during their period of dominance till the 7th century AD (Arik, 2002; Özgen, 2011). Until the 1960s, most of Hasankeyf s residents lived in ancient caves built into cliffs along the river. Today, some residents still live in the caves (Figure 2).

According to an archaeological study conducted in Hasankeyf Höyük, Hasankeyf was a hunter-gatherer settlement without ceramic ware. So far, the wild plant species found during the excavations in the region include almonds, pistachio, hackberry, lentil, and indeterminate nut species (Miyake et al., 2012). However, most archaeological sites in the region will be submerged by the construction of the Ilsu Dam, which is a part of the Southeast Anatolian Project [Güneydoğu Anadolu Projesi (GAP)], one of Turkey's largest hydroelectric projects to date (Çevik, 2012; Miyake et al., 2012). The water level of the dam 


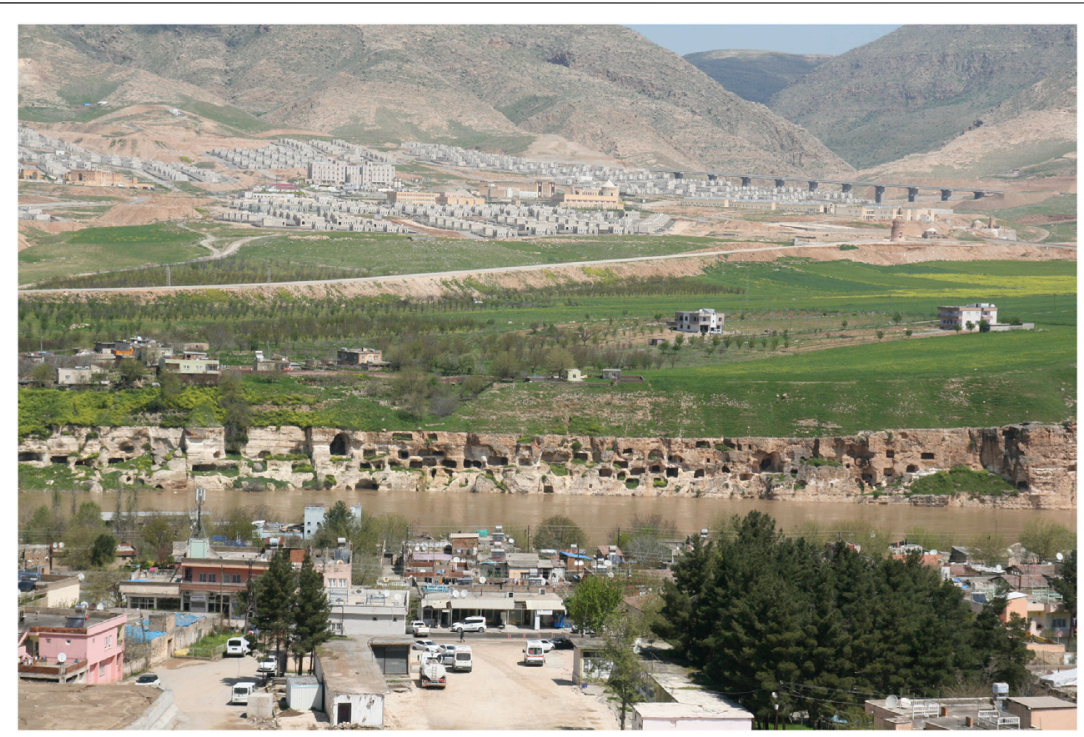

FIGURE 3 | The old and new settelements April 2019.

has raised the water level of the Tigris River, which now submerges more than $80 \%$ of the ancient city. Seven historical structures were moved to the new settlement. The inhabitants of the city center and three villages lost their land and livelihoods and as a result moved to the new settlement.

In a floristic study conducted in the Hasankeyf district center and its surroundings, 472 taxa belonging to 279 genera and 64 families were identified (Atamov et al., 2014). According to this study, 20 taxa are under threat because they are endemic and found only in this region. In addition, recently two plant species were identified in the sites of the ancient city and the Ilısu Dam: Onopordum hasankeyfense Pınar and Behçet (Pınar and Behçet, 2014) and Salvia hasankeyfense Dirmenci, Celep, and O. Güner (Celep et al., 2015). Populations of these species are at risk of extinction if conservation measures are not taken, and the species are in the category of Critically Endangered (CR) species.

In addition to historic places, plants, and other living things, traditional knowledge is also under threat of being lost in the research area due to the dam project. It is inevitable that ethnobotanical knowledge would be forgotten over time, especially due to the migration. It is therefore vital to conduct a detailed investigative research on the traditional ethnomedicinal knowledge of plants used in Hasankeyf and its surroundings. In our project design, we focused on: (1) compiling the ethnomedicinal knowledge of plants in the old and multicultural district; (2) frequently visiting the areas where intensive migration will be experienced due to the dam (Hasankeyf center and some villages); (3) spending sufficient time with the local people to obtain more intensive ethnobotanical knowledge; (4) comparing the obtained ethnomedicinal data with those of nearby regions; and (5) uncovering new ethnomedicinal uses of plants.

\section{MATERIALS AND METHODS}

\section{The Study Area}

Hasankeyf is located to the north of the Midyat and Raman hills (Özgen, 2011). The old city center was located $37 \mathrm{~km}$ Southeast of Batman city and generally had low lying hills (Özgen, 2011). The new settlement is located at the opposite slope of the old city, on the other side of the Tigris River (Figure 3).

The Hasankeyf region has an area of $529.95 \mathrm{sq} . \mathrm{km}$. There were 22 villages (Akalın, Aksu, Bayırlı, Büyükdere, Çardaklı, Gaziler, Güneşli, Irmak, İncirli, Karaköy, Kayıklı, Kelekçi, Kumluca, Öğütlü, Palamut, Saklı, Soğucak, Tepebaşı, Uzundere, Üçyol, Yakaköy, and Yolüstü) in rural areas and three settlements (Bahçelievler, Kale, and Eyyubi) in urban (Figures 4, 5); however, three settlements of the city center and three villages were flooded. The altitude is between 520 and 1,200 m. The Tigris River has an impact on the climate of the region, thus contributing to the area's mild winters (Batman Governorship, 2018). The annual average temperature is $25^{\circ} \mathrm{C}$. The highest average temperature is $40-43^{\circ} \mathrm{C}$ (July), while the lowest average temperature is $6-8^{\circ} \mathrm{C}$ (January). Precipitation primarily occurs in winter and spring, with an average annual rainfall of about $542 \mathrm{~mm}$ (Climate data for cities worldwide, 2012). This area belongs to the Irano-Turanian Plant Geography Region and falls within the C8 grid square according to the grid classification system developed by Davis (1965-1985). The dominant vegetation is steppe. There are small oak forests and maquis at an altitude of $800 \mathrm{~m}$ and above. The area is covered with small oak forests and maquis. Amygdalus orientalis, Amygdalus arabica Oliv., Celtis tournefortii, Crataegus azarolus L., Olea europaea Lindl., Pistacia species, Paliurus spina-christi, and Juniperus oxycedrus are among the important maquis species in this area (Atamov et al., 2014). 


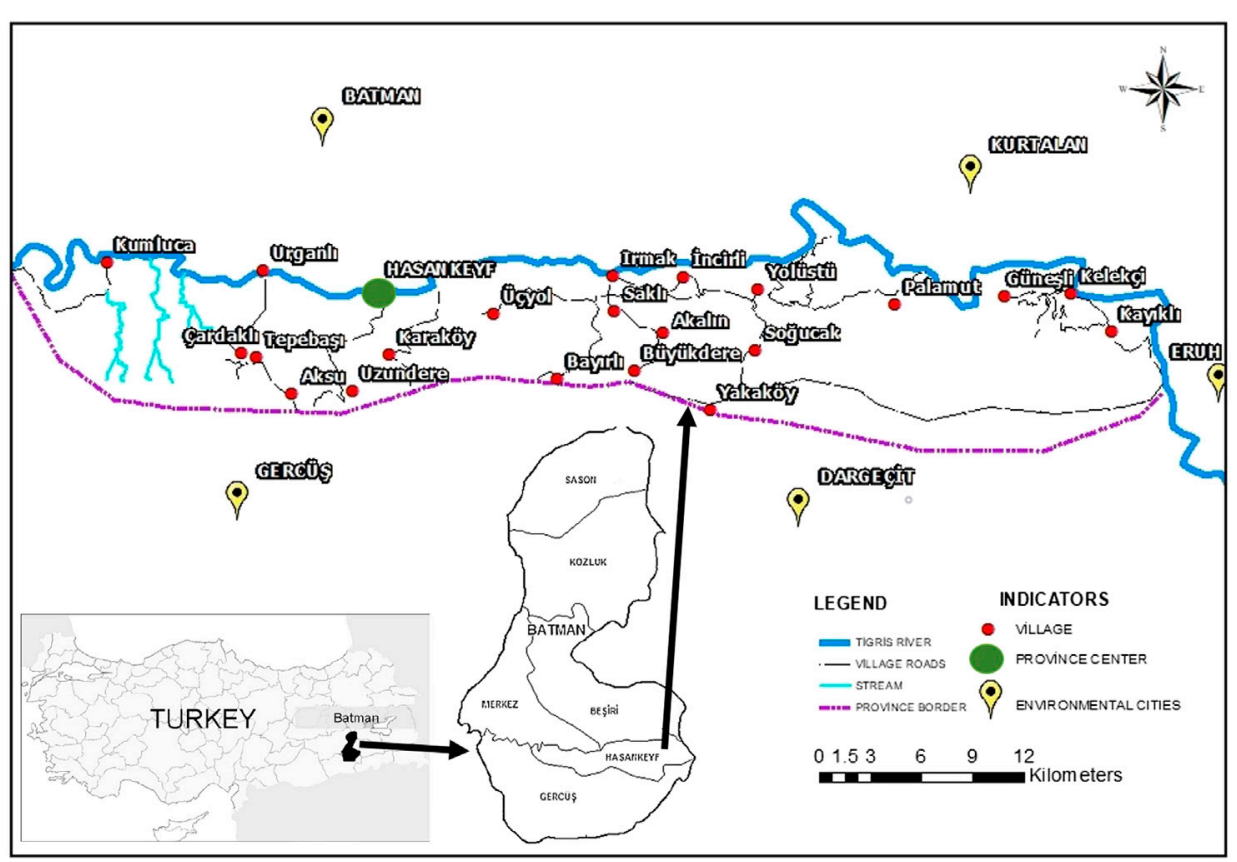

FIGURE 4 | The map of the study area, and its location in Batman Province and in Turkey.
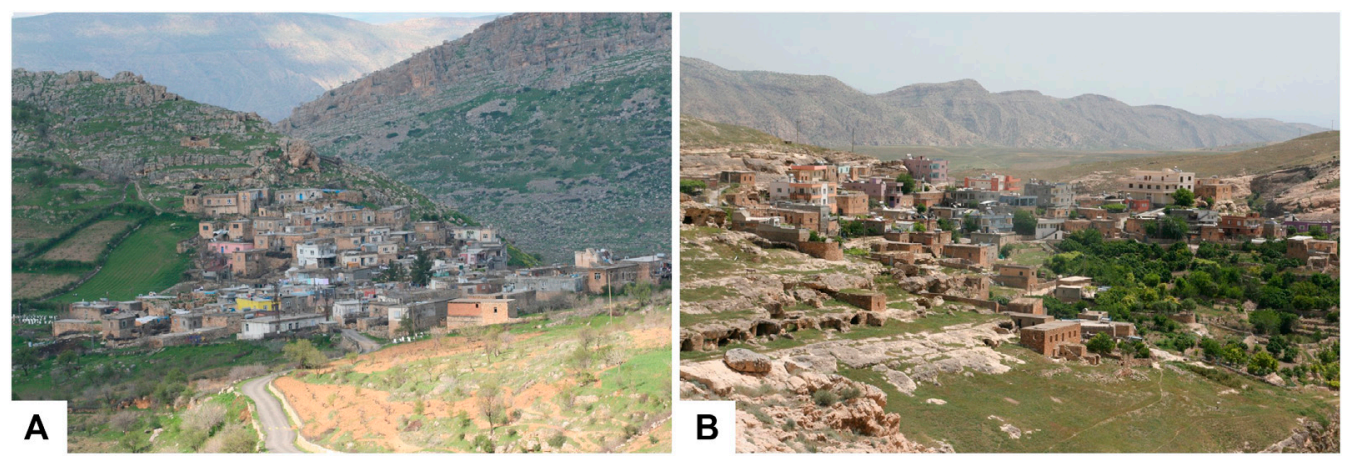

FIGURE 5 | Two villages of Hasankeyf (A) Karaköy (B) Üçyol.

Since it was an ancient city, the main source of livelihood of Hasankeyf was tourism in the city center. On the other hand, agriculture, horticulture, and livestock production were the main sources of income in the villages. Wheat is the main product of Hasankeyf, in addition to grape, almond, fig, lentil, and watermelon (Batman Governorship, 2018).

\section{Interviews With Local People}

A questionnaire was administered to the local people through face-to-face interviews (Appendix A).

The interviews were conducted with people in their homes, mosques, village squares, teahouses, gardens, or fields. Also, villages that would be submerged were visited more frequently. The official language in the study area is Turkish; however, most of the locals speak Arabic and Kurdish in Hasankeyf center, while the people who live in the villages generally speak Kurdish. The interviews were conducted in their mother tongues to ensure that they are able to express themselves comfortably. In the first year (2017), field studies were conducted by both authors. In the following 2 years (2018-2019), YY continued to study in the villages with the assistance of a local guide who speaks Kurdish and Turkish, and the interviews were conducted in Kurdish. At the town center, from the beginning of the study, the interviews were conducted in Arabic, Kurdish, and Turkish with the assistance of a local guide who can speak Arabic, Kurdish, and Turkish. For this reason, there was no problem of communication in the study area.

The Code of Ethics of the International Society of International Society of Ethnobiology (2008) was followed. The purpose of the study was explained to all participants and interviews were held only after they gave their approval to 
TABLE 1 | The categories of ailments and associated informant consensus factor (FIC) values.

\begin{tabular}{|c|c|c|c|}
\hline The ailment categories & Number of taxa & Use citations & FIC \\
\hline Internal and external inflammatory problems & 13 & 101 & 0.89 \\
\hline Diabetes & 12 & 64 & 0.82 \\
\hline Urogenital and kidney problems & 15 & 69 & 0.79 \\
\hline Respiratory diseases & 29 & 98 & 0.71 \\
\hline Rheumatic pain & 5 & 13 & 0.66 \\
\hline Ear disorders & 6 & 15 & 0.64 \\
\hline Blood problems: hypertension, hematinic, hemostatic & 7 & 17 & 0.62 \\
\hline Hemorrhoids & 6 & 14 & 0.61 \\
\hline Cardiac diseases & 3 & 6 & 0.60 \\
\hline Gastrointestinal disorders & 40 & 97 & 0.59 \\
\hline Snake bite, scorpion sting, fly bite, and parasitic disease & 4 & 8 & 0.57 \\
\hline Healing wounds, skin and hair care & 11 & 25 & 0.58 \\
\hline Anticancer & 3 & 5 & 0.50 \\
\hline Boosting the immune system & 3 & 5 & 0.50 \\
\hline Toothache & 6 & 12 & 0.45 \\
\hline Delighting, sedative, regulate the taste of the mouth & 5 & 8 & 0.42 \\
\hline Eye disorders & 7 & 11 & 0.40 \\
\hline
\end{tabular}

participate. Following the rules of Arabic and Kurdish language, local plant names were given in Latin alphabets.

\section{Plant Materials}

The field studies took place from March 2017 to November 2019. During this time, 171 plant specimens were collected. The plants were identified using the Flora of Turkey and the East Egean Islands (Davis, 1965-1985; Davis et al., 1988; Güner et al., 2000), A checklist of the flora of Turkey (vascular plants) (Güner et al., 2012), Illustrated flora of Turkey [vol. 1 (Güner, 2014) and vol. 2 (Güner et al., 2018)]. Plant specimens were deposited at the Herbarium of Istanbul University's Faculty of Pharmacy (ISTE). Plant parts obtained during the field studies are numbered with HSF (Hasankeyf) code and stored in envelopes and jars. The scientific names of the plant taxa were checked and controlled according to the checklist of the flora of Turkey (Güner et al., 2012) and The Plant List website (The Plant List, 2013).

\section{Ethnobotanical Indices Informant Consensus Factor (FIC)}

The FIC was originally developed by Trotter and Logan (1986) and then readapted by Heinrich (2000). The FIC value was used to analyze whether there was a consensus amongst the informants of the study area in the use of plants for various ailment categories. The FIC was calculated by the following formula: $\mathrm{FIC}=\mathrm{Nur}-\mathrm{Nt} / \mathrm{Nur}-1$, where Nur refers to the number of use citations in each category and $\mathrm{Nt}$ to the number of the species used. This measures the homogeneity of the ethnobotanical knowledge. FIC values range from 0 to 1. A high value indicates that informants are in agreement on the use of taxa for a category of illness. A low value indicates that plants are chosen randomly or informants do not exchange information about their uses. A high FIC indicates the informants' agreement about the taxa used for the treatment of ailments of a certain use category (Table 1).

\section{Use Value (UV)}

The relative importance of each species that the informants provided information for was found using the UV calculation. UV was calculated based on the following formula (Phillips et al., 1994):

$$
U V=\sum \frac{U}{N}
$$

Here, UV is the use-value of a species; $\mathrm{U}$ is the number of uses cited by each informant for a given plant species and $N$ is the total number of informants interviewed for a given plant. If the UV value is low, it indicates that the plant is not widely known. However, if the UV value is high, IT suggests that the plant is a frequently used, popular plant.

\section{Jaccard's Similarity Index}

A Venn diagram was drawn based on the medicinal taxa by the three selected regions and the related Jaccard Similarity Index for each pairing of the considered regions was calculated.

Jaccard's similarity index considers the similarity between two OTUs (operational taxonomic units) as the number of attributes shared divided by the total number of attributes present in either of the OTUs. Jaccard's index may be expressed as follows:

$$
\mathrm{J}=\mathrm{C} / \mathrm{A}+\mathrm{B}
$$

Here, $\mathrm{A}$ is the number of attributes present in OTU a, B is the number of attributes present in OTU b, and C is the number of attributes present in both OTUs a and $\mathrm{b}$. The number of attributes present in either of the OTUs is given by A + B (Jaccard, 1908).

\section{RESULTS}

\section{Demographic Features of the Interviewed Informants}

We interviewed a total of 131 informants, 76 females $(58.01 \%)$ and 55 males (41.98\%), whose age ranged from 8 to 98 years. 
TABLE 2 | Demographic details of the interviewed informants.

\begin{tabular}{|c|c|c|c|}
\hline \multirow{2}{*}{$\frac{\text { Categories }}{\text { Gender }}$} & \multirow[t]{2}{*}{ Subcategories } & \multicolumn{2}{|c|}{ Number of informants } \\
\hline & & Female & Male \\
\hline & & 76 & 55 \\
\hline \multirow[t]{7}{*}{ Age } & $8-21$ & 1 & 3 \\
\hline & $21-30$ & 8 & 4 \\
\hline & $31-40$ & 13 & 9 \\
\hline & $41-50$ & 14 & 9 \\
\hline & $51-60$ & 17 & 12 \\
\hline & $61-70$ & 19 & 12 \\
\hline & $71 \geq$ & 4 & 6 \\
\hline \multirow[t]{4}{*}{ Education level } & None & 35 & 9 \\
\hline & Primary & 28 & 23 \\
\hline & Secondary & 10 & 15 \\
\hline & Tertiary & 3 & 8 \\
\hline \multirow[t]{2}{*}{ Informant status } & Villages & 66 & 36 \\
\hline & City center & 10 & 19 \\
\hline \multirow[t]{5}{*}{ Employment status } & Retirement & - & 8 \\
\hline & Farmer & 20 & 34 \\
\hline & Housewife & 51 & - \\
\hline & Unemployed & 1 & 4 \\
\hline & Other jobs & 4 & 9 \\
\hline Total & & 131 & \\
\hline
\end{tabular}

Their education levels were varying-33.58\% having no education, $38.93 \%$ having primary education, $19.08 \%$ having secondary education, and only $8.39 \%$ having tertiary education. Most of them were Kurdish; thus, interviews with this group of informants were conducted in Kurdish and sometimes in Turkish. The female informants were less educated than males-26.71\% of female informants had not attended school, while only $6.87 \%$ of male informants had not attended school. Moreover, females aged 55 and over were not educated at all, especially those in rural areas. The demographic details of the informants are summarized in Table 2.

\section{General Figures and Most Represented Families and Species}

The plants utilized for medicinal purposes in the Hasankeyf district are listed in Table $\mathbf{3}$ and arranged in alphabetical order of their family and botanical names. In the course of this study, 171 specimens were collected and 94 taxa belonging to 72 genera and 40 families were recorded. Twenty families were represented by just one taxon, while the other 20 families were represented by 2 or more taxa. The predominant families were Lamiaceae (13), Asteraceae (8), Malvaceae (6), Rosaceae (6), Amaryllidaceae (5), Brassicaceae (4), Solanaceae (4), Boraginaceae (3), and Fabaceae (3) (Figure 6).

A total of 74 (78.72\%) wild species and $20(21.27 \%)$ cultivated species were recorded. Most taxa in this study were herbs (65), although there were a considerable number of shrubs (15 taxa) and trees (12 taxa). In addition, the use of one fern and one lichen were recorded in the study area.

We found that some wild medicinal plant taxa were widely used for commercial purposes. Celtis tournefortii (gengeres, taew, taav), Pistacia palaestina (bittım, qezwan), Pistacia eurycarpa (bittım, qezwan, benik), Quercus brantii (balot, baru beru), Rhus coriaria (sumaq, sımaq, tirş), Rosa canina (gulşîlan, gulşilav), Thymbra sintenisii (zahter, cahter, catir), Thymbra spicata (zahter, cahter), and Urtica dioica (gezgezok, gezo, gezgezk, qurrez) were the taxa extensively collected and marketed in the local bazaar and shops. These plants are collected mostly by women and provide a minor source of income for the local populace.

Several medicinal plants were reported as poisonous. It was explained to us that the latex should not come into contact with the tongue while using the latex of Euphorbia craspedia, Euphorbia macroclada, and Ficus carica species for amelioration of toothache. Also, it has been mentioned that Teucrium polium plant should be used in very small quantities, since an excess intake of it may cause irritation to the stomach. In addition, the hallucinogenic effect of Hyoscyamus albus leaves and the poisonous effect of Dioscorea communis roots were stated.

\section{Parts of Plant Used}

The aerial parts (39) of medicinal parts were the most frequently used parts in herbal drugs to cure diseases; however, many other parts were also utilized: fruits (23), leaves (22), underground parts (10), flowers and inflorescences (8), latex (4), seeds (4), branches (3), whole parts (2), barks (1), and resin (1) (Figures 7, 8). Sometimes, the local people also include other ingredients such as olive oil, animal milk, breast milk, honey, soap, salt, and sugar while preparing the remedies. In many cases, more than one organ of the same species is used in the preparation of different remedies.

\section{Methods of Drug Preparation and Utilization}

Various forms of drug preparation include infusion, decoction, fresh application, powdering, direct, chewing, crushing, paste, cooking, chewing, sucking, burning, maceration, and molasses. The most used forms of preparation of remedies are infusion (42), fresh application (25), crushing (16), powdering (10), and decoction (13). Also, the mostly utilized administration methods for preparations are internal (55), external (31), and eaten (22). Some species used for the treatment of earache were frequently mentioned. The powdered form of the plant is often mixed with breast milk, water, or soap and applied as ear drops. During the interviews, the informants stated specifically that the milk of a mother who had bore a baby girl should be used. There are different and interesting utilization methods for ear problems. For instance, the bulbs of Allium cepa are crushed and the obtained juice is mixed with soap and then applied as ear drops, or the fruits of Dioscorea communis are dried, powdered, and mixed with water and a teaspoon of it is applied as ear drops. Another utilization method is mixing the plant material with breast milk. As an example, the seeds of Persica vulgaris are powdered and mixed with breast milk before being applied as ear drops (three drops, two times a day until healed); the seeds of Hyoscyamus albus are powdered and mixed with breast milk before being applied as ear drops. Also, the fruits of Solanum americanum are dried, powdered, and burnt, and the affected ear is exposed to the smoke. 
TABLE 3 | Ethnomedicinal usage of the plants in Hasankeyf.

\begin{tabular}{|c|c|c|c|c|c|c|c|}
\hline Family & $\begin{array}{l}\text { Botanical name, } \\
\text { herbarium, } \\
\text { or collector number }\end{array}$ & Local name & $\begin{array}{c}\text { Plant part (s) } \\
\text { used }\end{array}$ & Preparation & $\begin{array}{l}\text { Utilization } \\
\text { method }\end{array}$ & $\begin{array}{c}\text { Therapeutic } \\
\text { effect/ailments treated }\end{array}$ & UV \\
\hline Adiantaceae & $\begin{array}{l}\text { Adiantum capillus-veneris } \\
\text { L. } \\
\text { ISTE } 116163\end{array}$ & $\begin{array}{l}\text { Pore fatme }(K) \text {, } \\
\text { şaar, cibbar }(A)\end{array}$ & Aerial parts & Infusion & Internal & $\begin{array}{l}\text { Diuretic, abdominal pain, } \\
\text { antitussive, cardiac diseases, } \\
\text { shortness of breath }\end{array}$ & 0.03 \\
\hline \multirow[t]{5}{*}{ Amaryllidaceae } & $\begin{array}{l}\text { Allium ampeloprasum L. } \\
\text { ISTE } 116272\end{array}$ & $\begin{array}{l}\text { Sîrik, sîrim, } \\
\text { sîrikapenir (K) }\end{array}$ & $\begin{array}{l}\text { Leaves } \\
\text { Bulbs }\end{array}$ & Fresh & $\begin{array}{l}\text { Eaten } \\
\text { External }\end{array}$ & $\begin{array}{l}\text { Antihypertensive } \\
\text { Eye burning }\end{array}$ & 0.04 \\
\hline & aAllium cepa $\mathrm{L}$. & Basal (A), pîvaz (K) & Bulb & $\begin{array}{l}\text { Fresh } \\
\text { Fresh } \\
\text { Onion juice with } \\
\text { soap, direct }\end{array}$ & $\begin{array}{l}\text { Eaten } \\
\text { External } \\
\text { Smelling } \\
\text { Dropped into } \\
\text { the ear }\end{array}$ & $\begin{array}{l}\text { Galactagogue, } \\
\text { Removing inflammations } \\
\text { caused by plant spines on the } \\
\text { skin and removing the spines } \\
\text { Respiratory tract } \\
\text { Ear pain }\end{array}$ & 0.29 \\
\hline & $\begin{array}{l}\text { Allium kharputense } \\
\text { Freyn and Sint. } \\
\text { ISTE } 115344\end{array}$ & $\begin{array}{l}\text { Sîrim, surım, } \\
\text { solyask, } \\
\text { sorelask (K) }\end{array}$ & Bulb, leaves & Crushed & $\begin{array}{l}\text { External (to the } \\
\text { eyelids) }\end{array}$ & Eye pain & 0.05 \\
\hline & ${ }^{\mathrm{a}}$ Allium sativum $\mathrm{L}$. & Sîr (K), ŝm, fum (A) & Bulbils & $\begin{array}{l}\text { Fresh } \\
\text { With salt } \\
\text { Added to olive oil }\end{array}$ & $\begin{array}{l}\text { Eaten } \\
\text { External } \\
\text { Internal }[1 \times(1) \\
2, \text { mh] } \\
\text { External }\end{array}$ & $\begin{array}{l}\text { Eye diseases, antihypertensive, } \\
\text { Toothache } \\
\text { Stomachache } \\
\text { Alopecia }\end{array}$ & 0.21 \\
\hline & $\begin{array}{l}\text { Allium scorodoprasum L. } \\
\text { ISTE } 116125\end{array}$ & $\begin{array}{l}\text { Sîrik, sîrim, } \\
\text { sîrimapenir (K) }\end{array}$ & Bulbils, leaves & Crushed & External & Eye burning & 0.03 \\
\hline \multirow[t]{3}{*}{ Anacardiaceae } & $\begin{array}{l}\text { Pistacia palaestina Boiss. } \\
\text { ISTE } 115361\end{array}$ & $\begin{array}{l}\text { Bittım }(A) \\
\text { qezwan }(K)\end{array}$ & Fruits & Crushed & $\begin{array}{l}\text { Prepared soap } \\
\text { External }\end{array}$ & Hair loss, hair care, skin care & 0.17 \\
\hline & $\begin{array}{l}\text { Pistacia eurycarpa Yalt. } \\
\text { ISTE } 115361\end{array}$ & $\begin{array}{l}\text { Bittım }(A) \text {, qezwan, } \\
\text { benik }(K)\end{array}$ & $\begin{array}{l}\text { Resin } \\
\text { Fruits }\end{array}$ & $\begin{array}{l}\text { Chewing } \\
\text { Crushed }\end{array}$ & $\begin{array}{l}\text { Internal } \\
\text { As coffee }\end{array}$ & $\begin{array}{l}\text { Lung and liver diseases } \\
\text { Delighting }\end{array}$ & 0.16 \\
\hline & $\begin{array}{l}\text { Rhus coriaria L. } \\
\text { ISTE } 115365\end{array}$ & $\begin{array}{l}\text { Sumaq, sımaq }(A \text {, } \\
K) \text {, tirş }(K)\end{array}$ & Fruits & Infusion & $\begin{array}{l}\text { Gargle } \\
\text { Internal }\end{array}$ & $\begin{array}{l}\text { Mouth sore } \\
\text { Antidiarrheic, antiemetic, } \\
\text { digestion problems }\end{array}$ & 0.23 \\
\hline \multirow[t]{2}{*}{ Apiaceae } & $\begin{array}{l}\text { aPimpinella anisum L. } \\
\text { HSF27 }\end{array}$ & Elison $(\mathrm{K})$ & Fruits & Infusion & Internal & Carminative (children) & 0.08 \\
\hline & $\begin{array}{l}\text { Scandix stellata Banks } \\
\text { and Sol. } \\
\text { ISTE } 115038\end{array}$ & Ziçirk (K) & Aerial parts & Fresh & Eaten & Digestion troubles & 0.03 \\
\hline \multirow[t]{2}{*}{ Araceae } & $\begin{array}{l}\text { Arum rupicola Boiss. } \\
\text { ISTE115336 }\end{array}$ & Kardî (K), nubê (A) & Leaves & $\begin{array}{l}\text { Cooked, dried } \\
\text { then cooked }\end{array}$ & Eaten & Antitussive, menstrual pain & 0.17 \\
\hline & $\begin{array}{l}\text { Biarum sp. } \\
\text { HSF19 }\end{array}$ & Lopka kocabatri (K) & $\begin{array}{l}\text { Dried } \\
\text { underground } \\
\text { parts }\end{array}$ & Powdered & Internal (1 ts) & Antidiarrheic, hemorrhoids & 0.04 \\
\hline \multirow[t]{8}{*}{ Asteraceae } & $\begin{array}{l}\text { Achillea aleppica DC. } \\
\text { ISTE } 117182\end{array}$ & $\begin{array}{l}\text { Kulilka maran, } \\
\text { kulilka zer (K) }\end{array}$ & Aerial parts & Infusion & $\begin{array}{l}\text { Internal }(1 \times 1 / \\
2, \mathrm{~h})\end{array}$ & Stomachache & 0.06 \\
\hline & $\begin{array}{l}\text { Achillea arabica Kotschy } \\
\text { ISTE117183 }\end{array}$ & $\begin{array}{l}\text { Kulilka maran, } \\
\text { kulilka zer }(\mathrm{K})\end{array}$ & Aerial parts & Infusion & $\begin{array}{l}\text { Internal }(1 \times 1 / \\
2, \mathrm{~h})\end{array}$ & Stomachache & 0.08 \\
\hline & $\begin{array}{l}\text { Chondrilla juncea L. } \\
\text { ISTE } 116120\end{array}$ & Giyayêbenuşta (K) & Latex of root & Fresh & Eaten & Liver troubles & 0.01 \\
\hline & $\begin{array}{l}\text { Cota altissima (L.) J. Gay } \\
\text { ISTE } 116117\end{array}$ & $\begin{array}{l}\text { Beybanuç, beybun } \\
(\mathrm{A}, \mathrm{K})\end{array}$ & Inflorescences & Infusion & Internal & $\begin{array}{l}\text { Abdominal pain, common cold, } \\
\text { antitussive }\end{array}$ & 0.08 \\
\hline & $\begin{array}{l}\text { Cota austriaca (Jacq.) } \\
\text { Sch. Bip. } \\
\text { ISTE } 116150\end{array}$ & $\begin{array}{l}\text { Beybanuç, beybun } \\
(\mathrm{A}, \mathrm{K})\end{array}$ & Aerial parts & Decoction & Internal & $\begin{array}{l}\text { Abdominal pain, common cold, } \\
\text { antitussive }\end{array}$ & 0,08 \\
\hline & $\begin{array}{l}\text { Cota tinctoria (L.) J. Gay } \\
\text { ISTE } 115673\end{array}$ & Giyayêzer (K) & Aerial parts & Infusion & $\begin{array}{l}\text { Internal }[1 \times(1) \\
2, \mathrm{~h}]\end{array}$ & $\begin{array}{l}\text { Cardiac diseases, shortness of } \\
\text { breath }\end{array}$ & 0.09 \\
\hline & $\begin{array}{l}\text { Echinops orientalis } \\
\text { Trautv. } \\
\text { ISTE } 116276\end{array}$ & Serteşik, sitrik (K) & $\begin{array}{l}\text { Aerial parts } \\
\text { Inflorescences }\end{array}$ & Direct & External (prick) & Bloodletting & 0.02 \\
\hline & $\begin{array}{l}\text { Matricaria aurea (Loefl.) } \\
\text { Sch. Bip. } \\
\text { ISTE } 115341\end{array}$ & $\begin{array}{l}\text { Beybanuç }(A, K) \text {, } \\
\text { giyayêseva, } \\
\text { gihaseva }(K)\end{array}$ & Aerial parts & Decoction & $\begin{array}{l}\text { Internal }[1 \times(1) \\
2, \mathrm{~h}]\end{array}$ & $\begin{array}{l}\text { Abdominal pain, menstrual } \\
\text { pain, antitussive (two times a } \\
\text { day), afterpains, common cold, } \\
\text { antitussive } \\
\quad \text { (Continued on following }\end{array}$ & age) \\
\hline
\end{tabular}


TABLE 3 | (Continued) Ethnomedicinal usage of the plants in Hasankeyf.

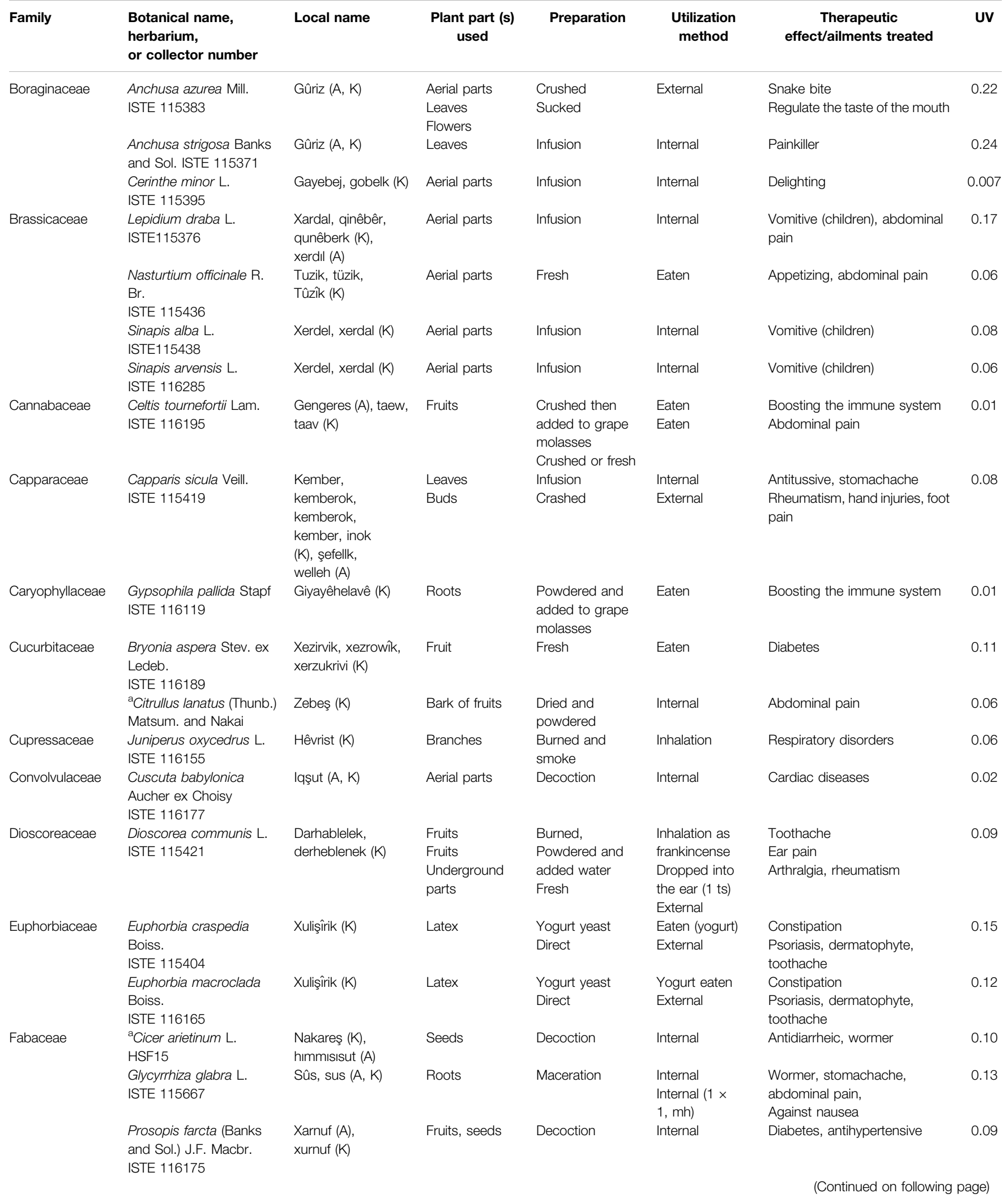


TABLE 3 | (Continued) Ethnomedicinal usage of the plants in Hasankeyf.

\begin{tabular}{|c|c|c|c|c|c|c|c|}
\hline Family & $\begin{array}{l}\text { Botanical name, } \\
\text { herbarium, } \\
\text { or collector number }\end{array}$ & Local name & $\begin{array}{l}\text { Plant part (s) } \\
\text { used }\end{array}$ & Preparation & $\begin{array}{l}\text { Utilization } \\
\text { method }\end{array}$ & $\begin{array}{c}\text { Therapeutic } \\
\text { effect/ailments treated }\end{array}$ & UV \\
\hline \multirow[t]{2}{*}{ Fagaceae } & $\begin{array}{l}\text { Quercus brantii Lindl. } \\
\text { ISTE } 115423\end{array}$ & $\begin{array}{l}\text { Balot }(A) \text {, baru, } \\
\text { beru }(K)\end{array}$ & $\begin{array}{l}\text { Fruits } \\
\text { Leaves }\end{array}$ & $\begin{array}{l}\text { Cooking in } \\
\text { embers or fresh } \\
\text { Infusion }\end{array}$ & $\begin{array}{l}\text { Eaten }[1 \times 3(4)] \\
\text { Internal }\end{array}$ & $\begin{array}{l}\text { Diabetes } \\
\text { Diabetes }\end{array}$ & 0.34 \\
\hline & $\begin{array}{l}\text { Quercus libani Oliv. } \\
\text { ISTE } 115422\end{array}$ & $\begin{array}{l}\text { Balot }(A) \text {, baru, } \\
\text { beru }(K)\end{array}$ & $\begin{array}{l}\text { Fruits } \\
\text { Leaves }\end{array}$ & $\begin{array}{l}\text { Fresh } \\
\text { Infusion }\end{array}$ & $\begin{array}{l}\text { Eaten }(1 \times 3, \mathrm{~h}) \\
\text { Internal }\end{array}$ & $\begin{array}{l}\text { Diabetes } \\
\text { Diabetes, antitussive, common } \\
\text { cold }\end{array}$ & 0.09 \\
\hline Hypericaceae & $\begin{array}{l}\text { Hypericum triquetrifolium } \\
\text { Turra } \\
\text { ISTE } 115426\end{array}$ & Botav $(\mathrm{K})$ & $\begin{array}{l}\text { Aerial parts } \\
\text { Flowers }\end{array}$ & $\begin{array}{l}\text { Infusion } \\
\text { Direct }\end{array}$ & $\begin{array}{l}- \\
\text { External }(1 \times \\
1, n)\end{array}$ & $\begin{array}{l}\text { Liver disorders, diabetes } \\
\text { Eye contours, eye pain }\end{array}$ & 0.25 \\
\hline \multirow[t]{13}{*}{ Lamiaceae } & $\begin{array}{l}\text { Cyclotrichium } \\
\text { leucotrichus } \\
\text { (Stapf ex Rech. F.) } \\
\text { Leblebici ISTE } 116168\end{array}$ & Rehan cebel $(A)$ & Aerial parts & Infusion & Internal & Antitussive & 0.03 \\
\hline & $\begin{array}{l}\text { Melissa officinalis L. } \\
\text { ISTE } 116136\end{array}$ & $\begin{array}{l}\text { Giyakî çolê, giyaye } \\
\text { tirş (K) }\end{array}$ & $\begin{array}{l}\text { Dried or fresh } \\
\text { leaves } \\
\text { Aerial parts }\end{array}$ & Infusion & $\begin{array}{l}\text { Internal } \\
\text { Internal }(1 \times 1 \\
1-2 \text { tgs })\end{array}$ & $\begin{array}{l}\text { Sedative } \\
\text { Headache, respiratory } \\
\text { disorders, abdominal pain }\end{array}$ & 0.06 \\
\hline & $\begin{array}{l}\text { Mentha longifolia (L.) L. } \\
\text { subsp. typhides (Briq.) } \\
\text { Harley } \\
\text { ISTE } 116164\end{array}$ & Pûng $(K)$, pûnge $(A)$ & $\begin{array}{l}\text { Dried or fresh } \\
\text { leaves } \\
\text { Leaves }\end{array}$ & $\begin{array}{l}\text { Infusion } \\
\text { Fresh }\end{array}$ & $\begin{array}{l}\text { External }(1 \times 1) \\
\text { Eaten }\end{array}$ & $\begin{array}{l}\text { Abdominal pain, shortness of } \\
\text { breath, halitosis, antitussive, } \\
\text { common cold, menstrual } \\
\text { disorders, infertility } \\
\text { Rheumatism } \\
\text { Halitosis }\end{array}$ & 0.37 \\
\hline & $\begin{array}{l}\text { Origanum vulgare L. } \\
\text { subsp. gracile (C. Koch) } \\
\text { Letsw. } \\
\text { ISTE } 116162\end{array}$ & $\begin{array}{l}\text { Rehan cebel }(A) \text {, } \\
\text { rehan }(K)\end{array}$ & Aerial parts & Infusion & Internal & Antitussives & 0.09 \\
\hline & $\begin{array}{l}\text { Salvia multicaulis Vahl. } \\
\text { ISTE } 115391\end{array}$ & $\begin{array}{l}\text { Rihan, giyaçaye, } \\
\text { Çaye gahye, } \\
\text { siraketin (K) }\end{array}$ & Fruits & Infusion & Internal & $\begin{array}{l}\text { Urinary problems, abdominal } \\
\text { pain, menstrual pain, bronchitis }\end{array}$ & 0.19 \\
\hline & $\begin{array}{l}\text { Salvia palaestina Benth. } \\
\text { ISTE } 116158\end{array}$ & Giyaçaye (K) & Aerial parts & Infusion & Internal & Abdominal pain, menstrual pain & 0.08 \\
\hline & $\begin{array}{l}\text { Scutellaria orientalis L. } \\
\text { subsp. bornmuelleri } \\
\text { (Hausskn. Ex Bornm.) } \\
\text { Edmondson } \\
\text { ISTE } 116124\end{array}$ & Giyatâl (K) & Aerial parts & $\begin{array}{l}\text { Dried, crush } \\
\text { added sugar }\end{array}$ & $\begin{array}{l}\text { Internal }(1 \times 1 \text {, } \\
\text { ts, h) }\end{array}$ & Diabetes & 0.03 \\
\hline & $\begin{array}{l}\text { Sideritis libanotica Labill. } \\
\text { ISTE117181 }\end{array}$ & $\begin{array}{l}\text { Qirşika şin, şirtik, } \\
\text { şirtika şin, giyayê } \\
\text { hêjire (K) }\end{array}$ & Aerial parts & Infusion & As tea & Abdominal pain, diabetes & 0.10 \\
\hline & $\begin{array}{l}\text { Teucrium chamaedrys L. } \\
\text { subsp. sinuatum (Celak.) } \\
\text { Rech. f. } \\
\text { ISTE } 115393\end{array}$ & Giyatâl $(K)$ & $\begin{array}{l}\text { Aerial parts, } \\
\text { leaves } \\
\text { Leaves }\end{array}$ & Infusion & $\begin{array}{l}\text { Internal }(1 \times 1) \\
\text { Internal }(1 \times w)\end{array}$ & $\begin{array}{l}\text { Diabetes, postnatal drip } \\
\text { Diabetes, urinary problems }\end{array}$ & 0.03 \\
\hline & $\begin{array}{l}\text { Teucrium polium L. } \\
\text { ISTE } 116130\end{array}$ & $\begin{array}{l}\text { Bojdank, giyatâl }(K) \text {, } \\
\text { cede }(A)\end{array}$ & Aerial parts & $\begin{array}{l}\text { Infusion } \\
\text { Decoction } \\
\text { Dried, powdered } \\
\text { Boiled, as paste }\end{array}$ & $\begin{array}{l}\text { Internal }(1 \times 1) \\
\text { Internal }(1 \times 1 \text {, } \\
\text { ts }) \\
\text { Internal }(1 \mathrm{ts}, \\
\text { mh) } \\
\text { External }\end{array}$ & $\begin{array}{l}\text { Diabetes, hemorrhoids } \\
\text { Abdominal pain, } \\
\text { Diabetes, urinary problems } \\
\text { Eye pain }\end{array}$ & 0.47 \\
\hline & $\begin{array}{l}\text { Thymbra sintenisii Bornm. } \\
\text { and Azn. } \\
\text { ISTE } 116194\end{array}$ & $\begin{array}{l}\text { Cahter, catir }(K) \text {, } \\
\text { zahter }(A)\end{array}$ & Aerial parts & Infusion & Internal $(1 \times 1)$ & $\begin{array}{l}\text { Afterpains, antitussive, } \\
\text { common cold, flu, abdominal } \\
\text { pain, stomachache }\end{array}$ & 0.19 \\
\hline & $\begin{array}{l}\text { Thymbra spicata L. } \\
\text { ISTE } 116206\end{array}$ & $\begin{array}{l}\text { Cahter }(\mathrm{K}) \text {, } \\
\text { zahter }(\mathrm{A})\end{array}$ & Aerial parts & Infusion & Internal $(1 \times 1)$ & $\begin{array}{l}\text { Afterpains, antitussive, } \\
\text { common cold, flu, abdominal } \\
\text { pain, stomachache }\end{array}$ & 0.17 \\
\hline & $\begin{array}{l}\text { Thymus kotschyanus } \\
\text { Boiss. and Hohen. } \\
\text { ISTE } 116205\end{array}$ & Cahter (K) & Leaves & Infusion & Internal $(1 \times 1)$ & $\begin{array}{l}\text { Abdominal pain, afterpains, } \\
\text { antitussive, common cold, flu, } \\
\text { stomachache, throat ache }\end{array}$ & 0.06 \\
\hline Lythraceae & $\begin{array}{l}\text { aPunica granatum L. } \\
\text { ISTE } 116133\end{array}$ & $\begin{array}{l}\text { Hînar (K), } \\
\text { rımman (A) }\end{array}$ & Flowers & Crushed & Eaten (mh) & Hemorrhoids, stomachache & 0.12 \\
\hline
\end{tabular}


TABLE 3 | (Continued) Ethnomedicinal usage of the plants in Hasankeyf.

\begin{tabular}{|c|c|c|c|c|c|c|c|}
\hline Family & $\begin{array}{l}\text { Botanical name, } \\
\text { herbarium, } \\
\text { or collector number }\end{array}$ & Local name & $\begin{array}{l}\text { Plant part (s) } \\
\text { used }\end{array}$ & Preparation & $\begin{array}{l}\text { Utilization } \\
\text { method }\end{array}$ & $\begin{array}{c}\text { Therapeutic } \\
\text { effect/ailments treated }\end{array}$ & UV \\
\hline \multirow[t]{6}{*}{ Malvaceae } & $\begin{array}{l}\text { Alcea setosa (Boiss.) Alef. } \\
\text { ISTE } 115363\end{array}$ & Hîro (K), xitmi (A) & $\begin{array}{l}\text { Aerial parts } \\
\text { Roots } \\
\text { Aerial parts }\end{array}$ & $\begin{array}{l}\text { Dried and } \\
\text { powdered added } \\
\text { to bath water } \\
\text { Dried and } \\
\text { crushed parts } \\
\text { with milk }\end{array}$ & $\begin{array}{l}\text { External } \\
\text { Internal }\end{array}$ & $\begin{array}{l}\text { Hair care, skin disorders } \\
\text { Throat ache, antitussive, } \\
\text { bronchitis }\end{array}$ & 0.28 \\
\hline & $\begin{array}{l}\text { Alcea digitata (Boiss.) } \\
\text { Alef. } \\
\text { ISTE } 116148\end{array}$ & Hîro (K), xitmi (A) & Aerial parts & $\begin{array}{l}\text { Infusion of dried } \\
\text { and crushed } \\
\text { parts with milk }\end{array}$ & Internal (mh) & $\begin{array}{l}\text { Throat ache, antitussive, } \\
\text { bronchitis }\end{array}$ & 0.10 \\
\hline & $\begin{array}{l}\text { Althaea cannabina L. } \\
\text { ISTE } 116157\end{array}$ & Hîro (K), xitmi (A) & Aerial parts & $\begin{array}{l}\text { Infusion of dried } \\
\text { and crushed } \\
\text { parts with milk } \\
\text { Infusion of dried } \\
\text { and crushed } \\
\text { parts with milk }\end{array}$ & $\begin{array}{l}\text { Internal (mh) } \\
\text { Internal }\end{array}$ & $\begin{array}{l}\text { Throat ache } \\
\text { Antitussive, bronchitis }\end{array}$ & 0.11 \\
\hline & $\begin{array}{l}\text { Malva neglecta Wallr. } \\
\text { ISTE } 115390\end{array}$ & $\begin{array}{l}\text { Tolık, tolik }(K) \\
\text { tolıkê }(A)\end{array}$ & Aerial parts & Decoction & Internal & Abdominal pain, common cold & 0.25 \\
\hline & $\begin{array}{l}\text { Malva nicaeensis All. } \\
\text { ISTE } 115412\end{array}$ & $\begin{array}{l}\text { Tolık, tolik }(K) \\
\text { tolıkê }(A)\end{array}$ & Aerial parts & Decoction & Internal & Abdominal pain, common cold & 0.18 \\
\hline & $\begin{array}{l}{ }^{\mathrm{a} T i l i a} \text { cordata Mill. } \\
\text { HSF10 }\end{array}$ & Ixlamur $(\mathrm{A}, \mathrm{K})$ & Inflorescences & $\begin{array}{l}\text { Decoction, } \\
\text { infusion }\end{array}$ & Internal & $\begin{array}{l}\text { Throat ache, antitussive, } \\
\text { bronchitis }\end{array}$ & 0.21 \\
\hline \multirow[t]{2}{*}{ Moraceae } & 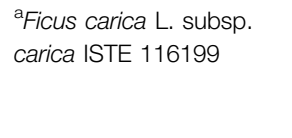 & $\begin{array}{l}\text { Hêjîr, hejîr (K), } \\
\text { tinê }(A)\end{array}$ & $\begin{array}{l}\text { Leaves } \\
\text { Fruit } \\
\text { Latex } \\
\text { Leaves }\end{array}$ & $\begin{array}{l}\text { Decoction } \\
\text { Fresh } \\
\text { Fresh }\end{array}$ & $\begin{array}{l}\text { External } \\
\text { Eaten } \\
\text { External }\end{array}$ & $\begin{array}{l}\text { Haemorrhoids, wart } \\
\text { Intestinal disorders } \\
\text { Toothache } \\
\text { Eye pain }\end{array}$ & 0.19 \\
\hline & $\begin{array}{l}\text { aMorus nigra L. } \\
\text { ISTE } 116149\end{array}$ & $\begin{array}{l}\text { Tuye reş, tuye } \\
\text { şemme }(K)\end{array}$ & Fruits & Fresh & $\begin{array}{l}\text { Eaten (h) } \\
\text { Eaten }\end{array}$ & $\begin{array}{l}\text { Wormer } \\
\text { Anticancer, hematinic }\end{array}$ & 0.06 \\
\hline Oleaceae & $\begin{array}{l}\text { aOlea europaea L. } \\
\text { HSF14 }\end{array}$ & $\begin{array}{l}\text { Zaytun }(A) \\
\text { zeytûn }(K)\end{array}$ & Leaves & Infusion & $\begin{array}{l}\text { Internal }(1 \times \\
1, \mathrm{mh})\end{array}$ & To defuse & 0.05 \\
\hline \multirow[t]{2}{*}{ Papaveraceae } & $\begin{array}{l}\text { Papaver glaucum } \\
\text { Boiss. and Hausskn. } \\
\text { ISTE } 115338\end{array}$ & $\begin{array}{l}\text { Xicxicok, xacxacok, } \\
\text { xecxecoka } \\
\text { şehika }(K)\end{array}$ & Aerial parts & $\begin{array}{l}\text { Crashed, as } \\
\text { paste }\end{array}$ & External & $\begin{array}{l}\text { Inflamed wound, abdominal } \\
\text { pain }\end{array}$ & 0.10 \\
\hline & $\begin{array}{l}\text { Papaver macrostomum } \\
\text { Boiss. and A. Huet } \\
\text { ISTE } 115082\end{array}$ & $\begin{array}{l}\text { Xicxicok, xacxacok, } \\
\text { xecxecoka } \\
\text { pîrçika }(K)\end{array}$ & Aerial parts & $\begin{array}{l}\text { Crushed, as } \\
\text { paste }\end{array}$ & External & $\begin{array}{l}\text { Inflamed wound, abdominal } \\
\text { pain }\end{array}$ & 0.06 \\
\hline Plantaginaceae & $\begin{array}{l}\text { Plantago major L. } \\
\text { ISTE } 117180\end{array}$ & $\begin{array}{l}\text { Palhavez, } \\
\text { pelhevez (K) }\end{array}$ & Leaves & Fresh & External & $\begin{array}{l}\text { Inflamed wound, } \\
\text { Removing inflammations } \\
\text { caused by plant spines on the } \\
\text { skin and removing the spines }\end{array}$ & 0.15 \\
\hline Platanaceae & aplatanus orientalis L. & Çinar (K) & Leaves & Infusion & Internal $(1 \times 2)$ & Rheumatism, arthrolith & 0.07 \\
\hline Poaceae & $\begin{array}{l}\text { aHordeum vulgare L. } \\
\text { ISTE } 115429\end{array}$ & $\mathrm{Ca}, \operatorname{ceh}(\mathrm{K})$ & Fruits & Decoction & Internal & Pass a kidney stone, diuretic & 0.08 \\
\hline Portulacaceae & $\begin{array}{l}\text { aportulaca oleracea L. } \\
\text { ISTE } 116209\end{array}$ & $\begin{array}{l}\text { Parparik pirpar }(K) \text {, } \\
\text { pirperê }(A)\end{array}$ & Aerial parts & Fresh & Eaten & Constipation & 0.05 \\
\hline \multirow[t]{2}{*}{ Ranunculaceae } & $\begin{array}{l}\text { Ranunculus cornutus DC. } \\
\text { ISTE } 115416\end{array}$ & Kunamella (K) & Aerial parts & Fresh, crushed & External & Inflamed wound & 0.13 \\
\hline & $\begin{array}{l}\text { Ranunculus millefolius } \\
\text { Banks and Sol. } \\
\text { ISTE } 116144\end{array}$ & Kulilke zer (K) & Aerial parts & Crushed & External & Inflamed wound & 0.09 \\
\hline Rhamnaceae & $\begin{array}{l}\text { Paliurus spina-christi Mill. } \\
\text { ISTE } 115346\end{array}$ & Driyesor, stiri (K) & $\begin{array}{l}\text { Fruits } \\
\text { Branches }\end{array}$ & $\begin{array}{l}\text { Crushed, added } \\
\text { to milk } \\
\text { Kept on fair, } \\
\text { flowing sap }\end{array}$ & $\begin{array}{l}\text { Internal } \\
\text { External }\end{array}$ & $\begin{array}{l}\text { Antitussive } \\
\text { Toothache }\end{array}$ & 0.06 \\
\hline
\end{tabular}

(Continued on following page) 
TABLE 3 | (Continued) Ethnomedicinal usage of the plants in Hasankeyf.

\begin{tabular}{|c|c|c|c|c|c|c|c|}
\hline Family & $\begin{array}{l}\text { Botanical name, } \\
\text { herbarium, } \\
\text { or collector number }\end{array}$ & Local name & $\begin{array}{l}\text { Plant part (s) } \\
\text { used }\end{array}$ & Preparation & $\begin{array}{l}\text { Utilization } \\
\text { method }\end{array}$ & $\begin{array}{c}\text { Therapeutic } \\
\text { effect/ailments treated }\end{array}$ & UV \\
\hline \multirow[t]{7}{*}{ Rosaceae } & $\begin{array}{l}{ }^{a} \text { Amygdalus communis L. } \\
\text { ISTE } 115435\end{array}$ & $\begin{array}{l}\text { Behîve tal, behîve } \\
\text { tal (K) }\end{array}$ & Fruits & Crushed & External & Mouth sore (children) & 0.14 \\
\hline & $\begin{array}{l}\text { Prunus orientalis (Mill.) } \\
\text { Koehne } \\
\text { ISTE } 116185\end{array}$ & Behîva eşk (K) & Fruits & Fresh & Eaten & Diabetes & 0.03 \\
\hline & aCydonia oblonga Mill. & Beh (K) & Fruits & Fresh & Inhalation & Sedative & 0.007 \\
\hline & $\begin{array}{l}\text { aPersica vulgaris Mill. (L.) } \\
\text { Batsch }\end{array}$ & Xox (K) & $\begin{array}{l}\text { Seeds } \\
\text { Leaves }\end{array}$ & $\begin{array}{l}\text { Powdered then } \\
\text { added to breast } \\
\text { milk } \\
\text { Infusion }\end{array}$ & $\begin{array}{l}\text { Dropped into } \\
\text { the ear }(1 \times 2,3 \\
\text { drs) } \\
\text { Dropped into } \\
\text { the ear }[1 \times(2) 3 \text {, } \\
3 \text { drs] }\end{array}$ & $\begin{array}{l}\text { Ear pain } \\
\text { Ear pain }\end{array}$ & 0.03 \\
\hline & Rosa canina L. & Gulşîlan, gulşilav (K) & Flowers & Infusion & Internal & Constipation & 0.17 \\
\hline & ISTE 116128 & & Fruits & Decoction & Internal & Abdominal pain, antitussive & \\
\hline & $\begin{array}{l}\text { Rubus sanctus Schreb. } \\
\text { ISTE } 115439\end{array}$ & $\begin{array}{l}\text { Awsâç }(A) \text {, dirîreşk, } \\
\text { dirîreşik, turêşk (K) }\end{array}$ & Fruits & Fresh & Eaten & Renal disorders hematinic & 0.08 \\
\hline Salicaceae & 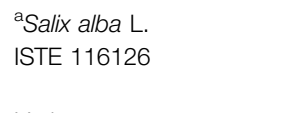 & $\mathrm{Bî}(\mathrm{K})^{3}$ & $\begin{array}{l}\text { Leaves } \\
\text { Branches, } \\
\text { barks }\end{array}$ & $\begin{array}{l}\text { Decoction } \\
\text { Infusion }\end{array}$ & Internal & $\begin{array}{l}\text { Antitussive } \\
\text { Antitussive, common cold, flu }\end{array}$ & 0.09 \\
\hline Scrophulariaceae & $\begin{array}{l}\text { Verbascum sp. } \\
\text { ISTE } 115420\end{array}$ & Balûtkefir (A) & Leaves & Crashed & External & Inflamed wound & 0.08 \\
\hline \multirow[t]{4}{*}{ Solanaceae } & $\begin{array}{l}\text { Hyoscyamus albus L. } \\
\text { ISTE } 115364\end{array}$ & $\begin{array}{l}\text { Benc, penc, } \\
\text { tûtinkosa, } \\
\text { tûtinkasa (K) }\end{array}$ & $\begin{array}{l}\text { Seeds } \\
\text { Leaves }\end{array}$ & $\begin{array}{l}\text { Powdered then } \\
\text { added to breast } \\
\text { milk } \\
\text { Fresh or } \\
\text { powdered } \\
\text { Crashed } \\
\text { Infusion }\end{array}$ & $\begin{array}{l}\text { Dropped into } \\
\text { the ear } \\
\text { External } \\
\text { External } \\
\text { Internal }\end{array}$ & $\begin{array}{l}\text { Ear pain } \\
\text { Inflamed wound, to remove } \\
\text { inflammation caused by spines } \\
\text { in the skin and to remove the } \\
\text { spines, athlete's foot, acne } \\
\text { Eye redness } \\
\text { Stomachache }\end{array}$ & 0.20 \\
\hline & $\begin{array}{l}\text { aLycopersicon } \\
\text { esculentum Mill. }\end{array}$ & Balcana sor (K) & Fruits & Fresh & External & Fly bite, scorpion sting & 0.03 \\
\hline & $\begin{array}{l}\text { Solanum americanum } \\
\text { Mill. } \\
\text { ISTE } 116154\end{array}$ & Kulike benav (K) & Fruits & $\begin{array}{l}\text { Dried, powdered, } \\
\text { burned }\end{array}$ & $\begin{array}{l}\text { Keeping the ear } \\
\text { over the smoke }\end{array}$ & Ear problems & 0.03 \\
\hline & asolanum tuberosum L. & Kartol (K) & Tubers & $\begin{array}{l}\text { Planed and } \\
\text { filtered juice }\end{array}$ & $\begin{array}{l}\text { Internal }(1 \times \\
1, \mathrm{mh})\end{array}$ & Stomachache & 0.03 \\
\hline Theaceae & $\begin{array}{l}{ }^{a} \text { Camellia sinensis (L.) } \\
\text { Kuntze }\end{array}$ & Çay (T) & Leaves & $\begin{array}{l}\text { Infusion, added } \\
\text { yogurt }\end{array}$ & Internal & Antidiarrheic & 0.08 \\
\hline \multirow[t]{2}{*}{ Urticaceae } & $\begin{array}{l}\text { Urtica dioica L. } \\
\text { ISTE } 115408\end{array}$ & $\begin{array}{l}\text { Gezgezok, gezo, } \\
\text { gezgezk }(K), \\
\text { qurrez }(A)\end{array}$ & Aerial parts & $\begin{array}{l}\text { Fresh } \\
\text { Infusion } \\
\text { Fresh } \\
\text { Fresh }\end{array}$ & $\begin{array}{l}\text { External } \\
\text { Internal }(1 \times 3) \\
\text { External } \\
\text { External }\end{array}$ & $\begin{array}{l}\text { Rheumatism, itching } \\
\text { Anticancer, anti-inflammatory } \\
\text { Hair loss } \\
\text { Hemostatic }\end{array}$ & 0.39 \\
\hline & $\begin{array}{l}\text { Urtica pilulifera L. } \\
\text { ISTE } 115339\end{array}$ & $\begin{array}{l}\text { Gezgezok, gezo, } \\
\text { gezgezk (K), } \\
\text { qurrez }(A)\end{array}$ & $\begin{array}{l}\text { Whole parts } \\
\text { Aerial parts }\end{array}$ & $\begin{array}{l}\text { Fresh } \\
\text { Infusion with fruit } \\
\text { of } R \text {. coriaria }\end{array}$ & $\begin{array}{l}\text { External } \\
\text { Internal }(1 \times 3) \\
\text { External }\end{array}$ & $\begin{array}{l}\text { Anti-inflammatory, rheumatism, } \\
\text { itching } \\
\text { Diabetes, anticancer, } \\
\text { antitussive } \\
\text { Hemostatic }\end{array}$ & 0.07 \\
\hline Vitaceae & $\begin{array}{l}\text { aVitis vinifera L. } \\
\text { ISTE } 115381\end{array}$ & $\begin{array}{l}\text { Anup }(A) \text {, mêw, tirîyi } \\
\text { mezruni }(K)\end{array}$ & Fruits & Molasses & Eaten & $\begin{array}{l}\text { Boosting the immune system, } \\
\text { antitussive }\end{array}$ & 0.04 \\
\hline Zygophyllaceae & $\begin{array}{l}\text { Tribulus terrestris L. } \\
\text { ISTE } 117179\end{array}$ & $\begin{array}{l}\text { Seddinank, } \\
\text { seddirank (K) }\end{array}$ & Aerial parts & Infusion & Internal $(1 \times 2)$ & $\begin{array}{l}\text { Diabetes, stomach disorders, } \\
\text { urinary disorders }\end{array}$ & 0.22 \\
\hline Stereocaulaceae & $\begin{array}{l}\text { Leptogium sp. } \\
\text { HSF20 }\end{array}$ & $\begin{array}{l}\text { Hinatirk, hennatitîk, } \\
\text { hınatitk }(\mathrm{K})\end{array}$ & Whole parts & Powder & External & $\begin{array}{l}\text { Dying hairs and hands, against } \\
\text { diaper rash, inflammation, } \\
\text { dermatophyte }\end{array}$ & 0.23 \\
\hline
\end{tabular}

${ }^{a}$ Cultivated plants.

Plant numbers: HSF, Hasankeyf; ISTE, Herbarium of Istanbul University's Faculty of Pharmacy.

Local names: A, Arabic; K, Kurdish; T, Turkish.

Utilization methods: drs, drops; $h$, hungry; mh, morning hungry; $n$, night; tgs, tea glasses; ts, teaspoon; $1 \times 1$, once a day; $1 \times(1) 2$, once a day or twice a day; $1 \times 2$, twice a day; $1 \times(2) 3$,

twice a day or three times a day; $1 \times 3$, three times a day; $1 \times 3$ (4), three times a day or four times a day; $1 \times w$, once a week.

Some mixtures are prepared for treatment. For instance, garlic, oil, and salt are mixed together and applied on the eyelid for alleviation of eye pain; henna and eggs are mixed together and applied to heal broken bones. Various uses of Matricaria aurea were recorded. It is mixed with Urtica species and Malva neglecta to treat abdominal pain. Furthermore, the infusion of Matricaria 


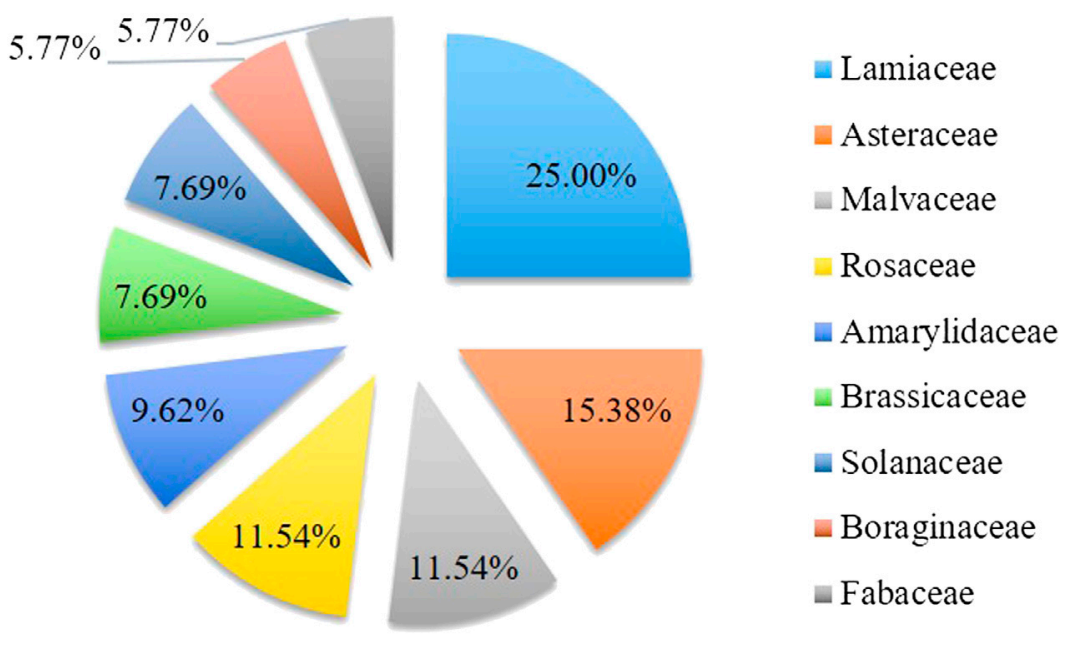

FIGURE 6 | The percentages of most used plant families in Hasankeyf.

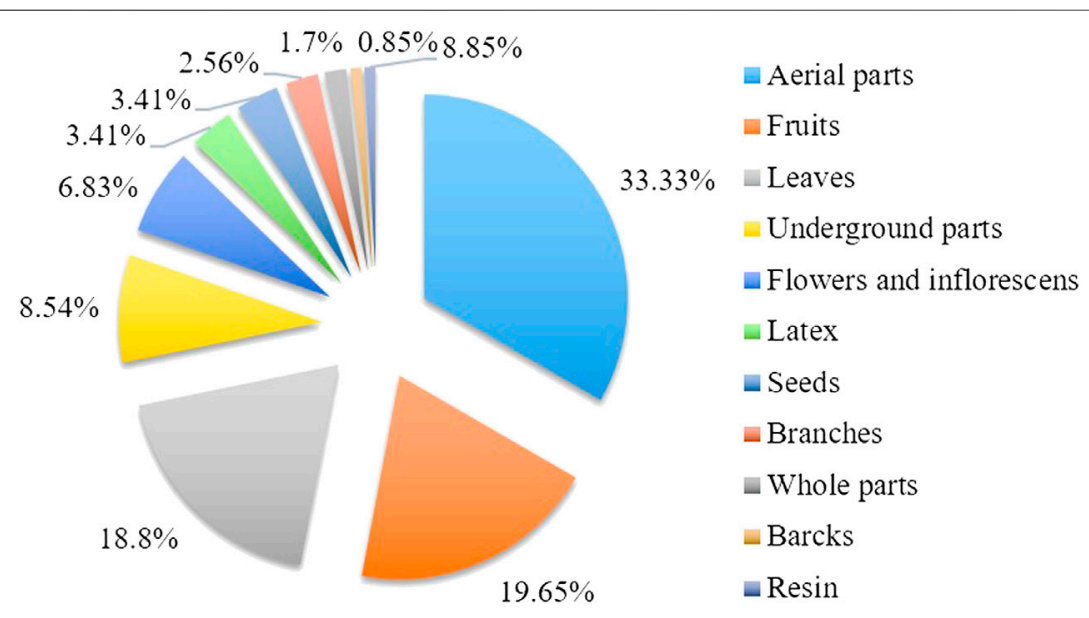

FIGURE 7 | The percentages of most used plant parts in Hasankeyf.
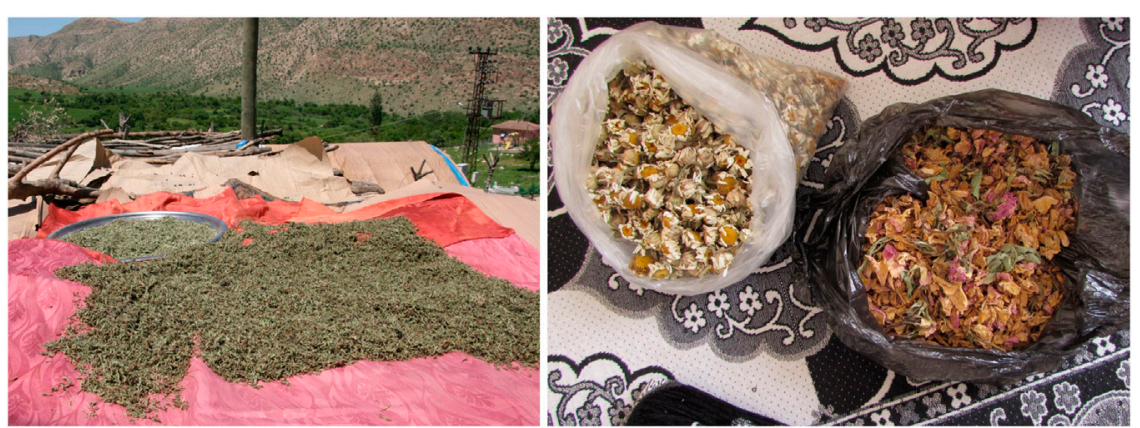

FIGURE 8 | Some medicinal plants used as dried in Hasankeyf.

aurea, Thymbra species, and Thymus species is used internally for the alleviation of menstrual pain and afterpains. Mentha longifolia has another use apart from the internal usage of aerial parts infusion. While this infusion is hot, it is poured into a basin and childless women stand on it for a while in a sitting position. This method is used to treat women with inflammation. 


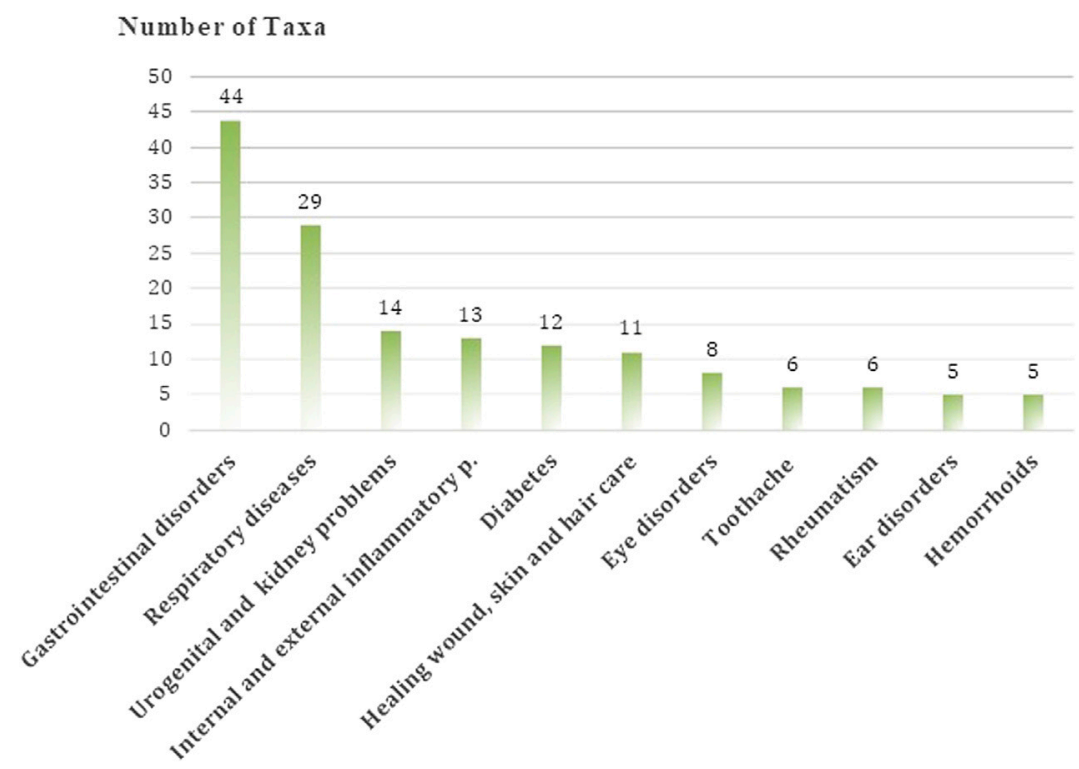

FIGURE 9 | The most common ailments treated by plants in Hasankeyf.

In addition, a mixture prepared with lemon juice, egg, and olive oil is used internally for the passing of kidney stones.

Crushed garlic bulbs are directly applied to previously blooded ringworm areas to treat ringworm.

\section{Ailments Treated by Plants}

The medicinal plants of Hasankeyf are used in the treatment of 69 different types of human ailments and diseases. Local people use herbal remedies most frequently for the treatment of gastrointestinal disorders (44), respiratory diseases (29), urogenital and kidney problems (14), internal and external inflammatory problems (13), diabetes (12), and to heal wounds, as well as for skin and hair care (11) (Figure 9).

\section{Calculations}

Ailments were grouped into 17 categories based on information gathered from the interviews (Table 3). Internal and external inflammatory problems had the highest FIC score (0.89). Allium cepa, Hyoscyamus albus, Plantago major, and Ranunculus species were among the plant remedies indicated for these problems. Diabetes was recorded to have the second highest FIC (0.82). Quercus brantii, Teucrium polium, Teucrium chamaedrys, and Bryonia aspera were among the plant remedies indicated for this use. Urogenital and kidney problems were recorded to have the third highest FIC (0.79), while respiratory diseases were recorded to have the fourth highest FIC value (0.76). Rheumatic pain was ranked as the fifth ailment, with an FIC value of 0.66 . An FIC value of 0.64 was recorded for ear disorders (Table 1).

According to UV analysis, species with the highest count of UV were Teucrium polium (0.47), Matricaria aurea (0.44), Urtica dioica (0.39), Mentha longifolia (0.37), Quercus libani (0.35), Allium cepa (0.29), Alcea setosa (0.28), Malva neglecta (0.25),
Hypericum triquetrifolium (0.25), Anchusa strigosa (0.24), Rhus coriaria (0.23), Lepraria finkii (0.23), Anchusa azurea (0.22), and Tribulus terrestris (0.22) (Table 3).

\section{DISCUSSION}

\section{Consumption of Medicinal Plants as Food}

A large proportion of medicinal plants are also being used as food in the region, thus indicating that the use of wild plants has a high potential in the area. About $49 \%$ (46 taxa) of the recorded medicinal plants in Hasankeyf are consumed in various ways as food. The wild medicinal plants investigated in this study are quite widely consumed raw as salad, pickles, preserves, and fruits. Also, they can be boiled and fried in a meal. Anchusa azurea, Anchusa strigosa, Malva neglecta, Nasturtium officinale, Urtica dioica, Urtica pilulifera, Papaver glaucum, Punica granatum, Quercus brantii, Rubus sanctus, Allium kharputens, Allium ampeloprasum, Allium scorodoprasum, and Celtis tournefortii were the medicinal plants most consumed as food in Hasankeyf.

Moreover, some medicinal plants were used as spices, especially in the rural areas. Cyclotrichium leucotrichum, Mentha longifolia subsp. typhoides, Origanum vulgare subsp. gracile, Rhus coriaria, Thymbra sintenisii, Thymbra spicata, and Thymus kotschyanus were used as spices.

In the study area, some medicinal plants were used as herbal tea, such as Melissa officinalis, Salvia multicaulis, and Thymbra spicata. In addition, Pistacia palaestina was consumed as coffee (Yeşil and İnal, 2019).

Since Arum rupicola is known for its toxic nature in the study area, local people subject it to a mandatory detoxification process before consuming it as medicine and 
food. This detoxification process has also been noted in previous ethnobotanical studies (Yeşil and Akalın, 2010; Pieroni et al., 2017; Pieroni et al., 2018; Yeşil and İnal, 2019; Yeşil et al., 2019; Kilıç et al., 2020).

In the study area, a syrup named "Gezo" was obtained from the leaves of the Quercus species by immersing them in a warm water spring. Thus, the concentrated sweet substance on the surface of the leaf is transferred into water. The obtained syrup is consumed as food when cooled and is also used as medicine for boosting the immune system; for treating cardiac diseases, stomach problems, diabetes; and for healing wounds. Furthermore, this syrup is added to molasses of grape and used for boosting the immune system. It is believed that Gezo fell down from the sky on oak trees, especially on to its leaves. It is produced by parasites of oak trees. The consumption of Gezo as food was stated previously by Gençay (2007) and Behçet and Arık (2013). Moreover, polyphenols analyzed by UHPLC-ESIMS/MS and antioxidant activities of molasses, acorn, and leaves of oak were studied, and it was found that Gezo molasses demonstrated higher scavenging effects than other extracts (Bursal et al., 2018).

There are more than 200 Allium species naturally distributed in Turkey and 30 of them have been used widely by the local people for various purposes as vegetable, spice, condiment, and medicine (Ekşi et al., 2020). In this study, it can be seen that five Allium taxa are used for medicinal purposes by the local people.

\section{Review of Local Plant Names}

The study area and the surrounding provinces comprise a multilingual population. When the plant names are generally examined, it was found that most of the plant names were in Kurdish and Arabic; only a few plant names were in Turkish.

Kozluk (Batman), Cizre (Şırnak), Artuklu (Mardin), and Midyat (Mardin) are close to the study area. Daisies are generally called beybun or beybanuç in the region. One of the most used plants Matricaria aurea is called giyayêseva, gihaseva, which means "apple herb" in Hasankeyf. The feature that distinguishes this plant from other daisies is the apple ("Sev" in Kurdish) scent of the plant. The plant is also known by the same name in Artuklu (Mardin) (Kilıç et al., 2020) and Midyat (Mardin) (Akgül et al., 2018).

On the other hand, the names of some local plants used in these areas are different. These local plants include Anchusa strigosa (hımhım), Cydonia oblonga (verekılfercel), Hypericum triquetrifolium (aran, ğırsile), Juniperus oxycedrus (difran), Malva neglecta (hibbes, tıbbayka, tabaknunu), Paliurus spinachristi (mağaylun), Salvia multicaulis (baravine, ikoro), Teucrium polium (cı̆̆de), and Tribulus terrestris (pıruğacuz) in Midyat (Mardin) (Akgül et al., 2018); Achillea aleppica (Kulilkamera, Isfaysara), Echinops orientalis (şekerok, şekirok), Melissa officinalis (pung, rihıtınneebune, nınhe), Salvia multicaulis (çaya çiyan, ikoro, bızzeyn), Salvia palaestina (çaya çiyan, ikoro, bızzeyn), Paliurus spina-christi (driya çalo, hezisk, mığeylen, sınc, selunê), and Tribulus terrestris (kurincok, korincok) in Artuklu (Midyat (Kilıç et al., 2020); Cyclotrichium leucotrichus (punge tata), Plantago major (belgeves), Salvia multicaulis (kaşketin), Salvia palaestina (ada çayı), Teucrium polium (merwend), and Tribulus terrestris (gurnig, kartıba) in Kozluk (Batman) (Bulut et al., 2019); and Prosopis farcta (hışhaş), Hypericum triquetrifolium (kantaron), Salvia multicaulis (giyacilık), and Teucrium chamaedrys (bojdank) in Cizre (Şırnak) (Gençay, 2007).

\section{Comparison of the Obtained Data With Those of Nearby Regions}

We calculated the similarity index of our study against other comprehensive studies conducted in nearby areas (Gençay, 2007; Yeşil and Akalın, 2009; Çakılcıŏlu and Türkoğlu, 2010; Tuzlacı and Doğan, 2010; Çakılcığlu et al., 2011; Polat et al., 2013; Kaval et al., 2014; Akgül et al., 2018; Polat, 2019; Kaya et al., 2020; Kılıç et al., 2020) and short-term ethnobotanical study of plants used for folk medicine in a nearby area (Bulut et al., 2019). The similarity index varied from 16.92 to $46.87 \%$. Maximum similarity was observed in the ethnobotanical study of plants in Midyat (Mardin). The first factor in this similarity is that Midyat is a close neighbor of Hasankeyf, and the second is they have been sharing similar habitats, flora, and similar social structures. The lowest index was obtained for Çatak (Van) (Mükemre et al., 2015), which was probably caused by the differences due to floral diversity and the characteristic habits of Çatak.

When we reviewed the plant families used for medicinal purposes in our study, Lamiaceae family emerged first. However, the Lamiaceae family emerged in first place only in the study conducted in Urfa (Kaya et al., 2020), while Asteraceae emerged first in all other studies compared. Furthermore, unlike other regions, we observed that plants in the Amaryllidaceae family are frequently used for various medicinal purposes in Hasankeyf.

In addition, the taxa not included in the studies presented in Table 4 are as follows Alcea digitata, Allium kharputense, Althaea cannabina, Dioscorea communis, Euphorbia craspedia, Hyoscyamus albus, Papaver glaucum, Ranunculus millefolius, and Scandix stellata. The medicinal purpose of these taxa were first recorded by this study in Hasankeyf and its surroundings.

In this study, it was observed that four of the five Allium species used for medicinal purposes in Hasankeyf were also used for the treatment of the ailments of the eye by the local populace. Furthermore, the use of Allium kharputense as food has been noted in previous studies (Gençay, 2007; Yeşil and Akalın, 2010; Arı et al., 2015; Firat, 2015; Yeşil and İnal, 2019). However, its medicinal use (for the treatment of eye pain) is recorded for the first time in Hasankeyf by this study. Antibacterial and antimicrobial activities of Allium species were stated in several studies (Fritsch and Keusgen, 2006; Panomket et al., 2012). Also, antimicrobial effect of methanolic extract from Allium kharputense has been previously determined (Erdoğan et al., 2015; İzol et al., 2020). 
TABLE 4 | The similarity percentages of studies in nearby areas.

\begin{tabular}{|c|c|c|c|c|c|}
\hline Citation & Location & $\begin{array}{l}\text { Number of } \\
\text { informants }\end{array}$ & $\begin{array}{c}\text { Total medicinal } \\
\text { taxa }\end{array}$ & $\begin{array}{c}\text { Common medicinal } \\
\text { taxa }\end{array}$ & $\begin{array}{c}\text { Similarity percentage } \\
(\%)\end{array}$ \\
\hline Akgül et al. (2018) & Midyat (Mardin) & 123 & 32 & 15 & 46.87 \\
\hline Çakılcıoğlu and Türkoğlu (2010) & Sivrice (Elazığ) & 176 & 81 & 21 & 25.92 \\
\hline Çakılcıoğlu et al. (2011) & Maden (Elazığ) & 143 & 88 & 21 & 23.86 \\
\hline Gençay (2007) & Cizre (Sirnak) & 60 & 44 & 19 & 43.18 \\
\hline Kaval et al. (2014) & Geçitli (Hakkâri) & 146 & 70 & 12 & 17.14 \\
\hline Kaya et al. (2020) & Urfa & 195 & 37 & 14 & 37.73 \\
\hline Kilıç et al. (2020) & Artuklu (Mardin) & 365 & 85 & 32 & 37.64 \\
\hline Polat et al. (2013) & Solhan (Bingöl) & 145 & 82 & 25 & 30.48 \\
\hline Polat (2019) & Bingöl & 182 & 93 & 24 & 25.80 \\
\hline Tuzlacı and Doğan (2010) & Ovacık (Tunceli) & - & 65 & 11 & 16.92 \\
\hline Yeşil and Akalın (2009) & Kürecik (Malatya) & 120 & 47 & 13 & 27.65 \\
\hline
\end{tabular}

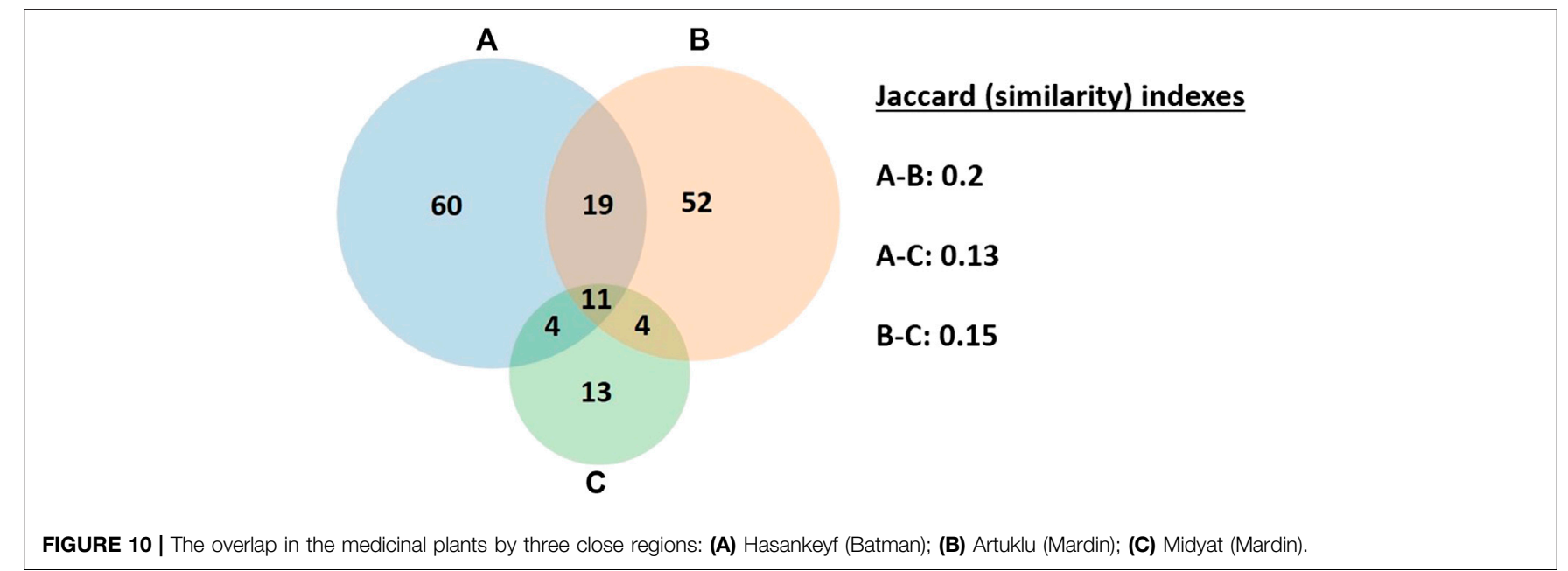

Medicinal use of plants from the Solanaceae family was not observed in ethnobotanical studies conducted in the regions close to Hasankeyf (Gençay, 2007; Polat, 2019; Yeşil and Akalın, 2009; Çakılcığlu and Türkoğlu, 2010; Tuzlacı and Doğan, 2010; Çakıllcığlu et al., 2011; Polat et al., 2013; Kaval et al., 2014; Akgül et al., 2018; Bulut et al., 2019; Kaya et al., 2020; Kılıç et al., 2020). The use of tobacco was recorded by Gençay (2007) for pleasure. Since its use is common, it may not be included in other studies. In addition, medicinal use of some members of the Solanaceae family is known in the eastern Anatolian region (Altundağ and Öztürk, 2011). Nevertheless, Solanum americanum has never been known for medicinal use in the East and Southeast of Turkey. In the study area, the smoke of burnt fruit of Solanum americanum is used for curing ear problems. As mentioned in a recent study (Afroz et al., 2020), Solanum genus contains plenty of snakin-2 peptide, which has significant antimicrobial activity. Also, there are four more taxa used for ear problems: Allium cepa, Dioscorea communis, Hyoscyamus albus, and Persica vulgaris. However, Dioscorea communis, Hyoscyamus albus, and Persica vulgaris were not mentioned in previous studies conducted in Eastern Turkey.
When this study is compared with other studies in terms of the number of informants and the number of medicinal taxa, it is clearly seen that the residents of Hasankeyf use traditional knowledge more efficiently. The reasons for this may be its long-standing background, its multicultural population, and its inhabitants' being in touch with nature and the diversity of flora.

A Venn diagram showing the overlap among Hasankeyf and two nearby regions (number of cited taxa) with high similarity percentages and the related Jaccard Similarity Indexes are presented in Figure 10. The most overlap of the obtained data and the Jaccard index was between Hasankeyf and Artuklu (Kiliç et al., 2020), which may be due to the similarity of the density of Kurdish and Arab populations (Muslims) in Artuklu and Hasankeyf. The least overlap, on the other hand, was between Hasankeyf and Midyat (Akgül et al., 2018), and the major reason for that seems to be that Midyat has a multireligious population. Ten taxa are used in common in all three regions: Anchusa azurea, Capparis sicula, Hypericum triquetrifolium, Malva neglecta, Matricaria aurea, Teucrium polium, Thymbra sintenisii, Tribulus terrestris, Paliurus spina-christi, and Urtica dioica. These taxa are culturally important plants in all three 
regions as they are used for different purposes (food, ornament, fuel, etc.) besides their medicinal use.

The medicinal use of Matricaria aurea species was only recorded in Hasankeyf, Midyat (Mardin) (Akgül et al., 2018), and Artuklu (Mardin) (Kılıç et al., 2020) regions. This species was the second plant with the highest UV in Hasankeyf and was frequently mentioned by women. In a previous study, it was stated that essential oils of Matricaria aurea are very rich in chemical compounds, especially phenolic-containing coumarin products, which have contributed to their antioxidant and antibacterial activity (Kheder et al., 2014).

The use of fruits of Paliurus spina-christi for toothache has been previously recorded (Tuzlac1, 2016). However, the use of the greasy material (obtained by burning the branches of the plant) for toothache was recorded for the first time in this study.

It was mentioned that the inflorescences of the Echinops orientalis plant directly struck the body to eliminate excess blood (bloodletting). Bloodletting, the practice of letting blood out to cure a patient, was for centuries one of the main therapies in the West (Miton et al., 2015). The use of E. orientalis for an ancient treatment method shows that the residents of Hasankeyf had impressive traditional knowledge.

\section{CONCLUSION}

In this study, the use of 94 plant taxa for 69 different types of ailments and diseases was determined in the Hasankeyf district. As a result of interviews with the individuals, it was observed that the plants frequently recorded in questionnaires include Teucrium polium, Matricaria aurea, Urtica dioica, Mentha longifolia Quercus brantii, Allium cepa, Alcea setosa, Malva neglecta, Hypericum triquetrifolium, Anchusa strigosa, Rhus coriaria, Lepraria finkii, Anchusa azurea, and Tribulus terrestris. These plants also have a widespread usage in the area and higher UVs. Also, the data indicate that the main illnesses treated by medicinal plants in Hasankeyf are gastrointestinal disorders, respiratory diseases, urogenital and kidney problems, internal and external inflammatory problems, diabetes, healing of wounds, as well as skin and hair care. Moreover, the treatment of ear problems, of which there are few records in ethnobotanical studies, has been frequently mentioned in the study area, and the uses and use methods of plants are stated by both young and old individuals. In addition, the method of bloodletting by the spins of a plant, which has an ancient history in the study area, is not recorded in ethnobotanical studies in the East and Southeast regions of Turkey. Taxa such as Allium kharputense, Althaea cannabina, Dioscorea communis, Euphorbia craspedia, Hyoscyamus albus, Papaver glaucum, Ranunculus millefolius, and Scandix stellata were not recorded in nearby areas before.

It was observed that individuals living in rural areas have more traditional knowledge about the use of medicinal plants than those living in urban areas. In addition, it was observed that women were more experienced in using plants compared to men; for instance, women aged 51 and over had knowledge about preparation and utilization methods of medicinal herbs in rural areas. Since the older individuals living in villages and individuals who have traditional knowledge are not educated, they speak only Kurdish; thus, they are not exposed to both TV and social networks (Kurdish is not used commonly in these mediums). However, this disadvantage turned into an advantage because such traditional knowledge is not contaminated with external information and is therefore undilutedly passed on from generation to generation. On the other hand, the knowledge is transmitted orally, which may lead to loss or at least deformation of information by time. At this point, this study is of great importance as this valuable knowledge is recorded.

Although, men in urban areas generally had knowledge about medicinal plants, detailed plant uses were mostly explained by women. Moreover, it has been observed that individuals in urban areas aged 61 and over had knowledge about medicinal plants. It is inevitable that the traditional use of plants will rapidly disappear from the cities and some villages due to migration to modern centers where lifestyles are completely different (livelihood, gathering food, receiving health care, new people, $\mathrm{TV}$, language, etc.)

The obtained traditional knowledge is an important resource for sustainable development in the study area. In particular, by establishing small-scale local cooperatives, it can be ensured that commonly used medicinal plants (Matricaria aurea, Hypericum triquetrifolium, Salvia multicaulis) are produced under suitable conditions and sold in the public market or in local shops in the region. In addition, some culturally important plants (Anchusa speies, Glycyrrhiza glabra, Pistacia palaestina, Thymbra species) can be grown in the region and these can be prepared and served in local restaurants using traditional methods. Furthermore, in the light of the obtained knowledge, it was observed that there were some plants that can be used to boost the immune system and to treat symptoms similar to that of Covid-19. However, as there are no enough previous studies, the chemical contents and activities of these plants should be investigated in detail.

Another significance of this study is that the obtained data may be an important and meaningful source for future ethnobotanical studies in Southeastern Turkey, as there have been very few studies carried out on plants due to local problems encountered in the region.

\section{DATA AVAILABILITY STATEMENT}

The raw data supporting the conclusions of this article will be made available by the authors, without undue reservation, to any qualified researcher.

\section{ETHICS STATEMENT}

Ethical review and approval was not required for the study on human participants in accordance with the local legislation and 
institutional requirements. The patients/participants provided their written informed consent to participate in this study. Written informed consent was not obtained from the individual(s) for the publication of any potentially identifiable images or data included in this article.

\section{AUTHOR CONTRIBUTIONS}

YY and İI carried out fieldwork, collected the data, and identified the plants in 2017, after which YY carried out the remaining fieldwork up to November 2019 YY analyzed the data and wrote the manuscript.

\section{REFERENCES}

Afroz, M., Akter, S., Ahmed, A., Rouf, R., Shilpi, J. A., Tiralongo, E., et al. (2020). Ethnobotany and antimicrobial peptides from plants of the Solanaceae family: an update and future prospects. Front. Pharmacol. 11, 565. doi:10.3389/fphar. 2020.00565

Ahunday, Z., and Balkız, Ö. (2009). Outstanding universal value of Hasankeyf and the Tigris valley, Doğa derneği. Available at: https://goo.gl/qZ9tqX (Accessed September 12, 2020).

Akan, H., Aydoğdu, M., Korkut, M. M., and Balos, M. M. (2013). An ethnobotanical research of the Kalecik mountain area (Sanliurfa, south-east Anatolia). Biol. Divers. Conserv. 6 (2), 84-90.

Akgül, A., Akgül, A., Senol, S. G., Yildirim, H., Seçmen, O., and Dogan, Y. (2018). An ethnobotanical study in Midyat (Turkey), a city on the silk road where cultures meet. J. Ethnobiol. Ethnomed. 14, 12. doi:10.1186/s13002-017-0201-8

Altundag, E., and Ozturk, M. (2011). Ethnomedicinal studies on the plant resources of east Anatolia, Turkey. Proc. Soc. Behav. Sci. 19, 756-777. doi:10.1016/j.sbspro.2011.05.195

Arı, S., Temel, M., Kargığlu, M., and Konuk, M. (2015). Ethnobotanical survey of plants used in Afyonkarahisar-Turkey. J. Ethnobiol. Ethnomed. 11 (1), 84. doi:10.1186/s13002-015-0067-6

Arık, M. O. (2002). "Hasankeyf 2000 studies," in The research of the archaeological and cultural heritage that will remain under the Ilısu and Karkamış dam lakes, 2000 (Ankara,Turkey: METU Historical Environment Research and Evaluation Center Publications), 773-778. (in Turkish).

Atamov, V., Çetin, E., Aslan, M., and Cevher, C. (2014). The flora of Ilısu/Turkey (Hasankeyf) and its conservation. Biol. Divers. Conserv. 7 (1), 16-31.

Batman Governorship (2018). Hasankeyf. Available at: http://batman.gov.tr/ ilcelerimiz/hasankeyf (Accessed September 15, 2020).

Baykal, H., and Atamov, V. (2017). Ethnobotanical documentation of plants of başhemşin valley, Kaçkar mountains national park, rize, Turkey. Bangladesh J. Bot. 46 (2), 767-773.

Behçet, L., and Arık, M. (2013). An ethnobotanical investigation in East Anatolia (Turkey). Turk. J. Nat. Sci. 2 (1), 1-14.

Bulut, G., Doğan, A., Şenkardeş, I., Avci, R., and Tuzlaci, E. (2019). The medicinal and wild food plants of Batman City and Kozluk district (Batman-Turkey). Agric. Conspec. Sci. 84 (1) 29-36.

Bulut, G., Haznedaroğlu, M. Z., Doğan, A., Koyu, H., and Tuzlac1, E. (2017). An ethnobotanical study of medicinal plants in Acipayam (Denizli-Turkey). J. Herb. Med. 10, 64-81. doi:10.1016/j.hermed.2017.08.001

Bursal, E., Bulut, G., and Boğa, R. (2018). Polyphenols analysed by UHPLC-ESIMS/ MS and antioxidant activities of molasses, acorn and leaves of oak (Quercus robur subsp. pedunculiflora). Prog. Nutr. 20 (1), 167-175. doi:10.23751/pn. v20i1-S.5311

Cakilcioglu, U., Khatun, S., Turkoglu, I., and Hayta, S. (2011). Ethnopharmacological survey of medicinal plants in Maden (Elazig-Turkey). J. Ethnopharmacol. 137, 469-486. doi:10.1016/j.jep.2011.05.046

Cakilcioglu, U., and Turkoglu, I. (2010). An ethnobotanical survey of medicinal plants in Sivrice (Elazığ-Turkey). J. Ethnopharmacol. 132, 165-175. doi:10. 1016/j.jep.2010.08.017

\section{FUNDING}

This work has been supported by the Istanbul University Scientific Research Projects Department under project TYD-2017-25239.

\section{ACKNOWLEDGMENTS}

Special thanks to Mahmut Inal, Bahattin Yılmaz, Hamza Yilmaz, Ömer Güzel, Kenan Yeşil, and Abdullah Olgun for helping us with field work; Nezir Gülçek for providing us with the map of Hasankeyf; Abdullah Kandemir for the photograph of Hasankeyf center (2013); and Ali Cantürk for reading the manuscript during the revision.

Celep, F., Dirmenci, T., and Güner, Ö. (2015). Salvia hasankeyfense (Lamiaceae), a new species from Hasankeyf (Batman, south-eastern Turkey). Phytotaxa 227 (3), 289-294. doi:10.11646/phytotaxa.227.3.9

Çelik, B., and Yeșil, Y. (2020). An ethnobotanical study in Pöhrenk village (Çiçekdağ1-Kırşehir province/Turkey). Ijp 50 (2), 131-141. doi:10.26650/ IstanbulJPharm.2019.0072

Çevik, A. (2012). Hasankeyf: the capital of civilizations. Doğa derneği [internet]. Available at: http://www.dogadernegi.org./wp-content/uploads/2015/10/ Hasankeyf-The-Capital-of-Civilizations (Accessed April 26, 2019).

Climate data for cities worldwide (2012). Batman. Available at: https://tr.climatedata.org/asya/tuerkiye/batman/batman-284/ (Accessed September 10, 2020).

Davis, P. H. (1965-1985). Flora of Turkey and the east Aegean Islands. Edinburgh, Scotland: Edinburg University Press, 1-9.

Davis, P. H., Mill, R. R., and Tan, K. (1988). Flora of Turkey and the east Aegean Islands. Edinburgh, Scotland: Edinburgh University Press, Vol. 10.

Ekşi, G., Gençler Özkan, A. M., and Koyuncu, M. (2020). Garlic and onions: an eastern tale. J. Ethnopharmacol. 253, 112675. doi:10.1016/j.jep.2020.112675

Erdogan, E., Yabalak, E., Everest, A., and Gizir, A. (2015). Mutagenic and antimicrobial evaluation of methanol extract from Allium kharputense Freyn Et. Sint. Spatula DD 5 (2), 83-87. doi:10.5455/spatula. 20151124063207

Ertuğ, F., and Güner, A. (2014). “Ethnobotany,” in Illustrated flora of Turkey (Istanbul, Turkey: Isbank Culture Publications), 1, 319-344. (in Turkish).

Fritsch, R. M., and Keusgen, M. (2006). Occurrence and taxonomic significance of cysteine sulphoxides in the genus Allium L. (Alliaceae). Phytochemistry 67 (11), 1127-1135. doi:10.1016/j.phytochem.2006.03.006

Firat, M. (2015). The ethnobotanical usage of some east Anatolian (Turkey) Allium L. species. Manas J. Agric. Life Sci. 5, 80-86.

Gençay, F. (2007). Cizre (Şırnak)'nin etnobotanik özellikleri [Ethnobotanical aspects of Cizre (Şırnak). MSc thesis/dissertation. Van, Turkey: Yüzüncü Yıl University Department of Biology.

Güler, B., Erkan, Y., and Uğurlu, E. (2020). Traditional uses and ecological resemblance of medicinal plants in two districts of the Western Aegean Region (Turkey). Environ. Dev. Sustain. 22, 2099-2120. doi:10.1007/s10668018-0279-8

Günbatan, T., Gürbüz, İ., and Gençler Özkan, A. M. (2016). The current status of ethnopharmacobotanical knowledgein Çamlıdere (Ankara, Turkey)*. Turk. J. Bot. 40, 241-249. doi:10.3906/bot-1501-37

Güner, A. (2014). Illustrated flora of Turkey. Istanbul, Turkey: Isbank Culture Publications, Vol. 1 (in Turkish).

Güner, A., Aslan, S., Ekim, T., Vural, M., and Babaç, M. T. (2012). A checklist of the Flora of Turkey (vascular plants). Istanbul, Turkey: Nezahat Gökyiğit Botanical Garden and Flora Research Association Publication (in Turkish).

Güner, A., Kandemir, A., Menemen, Y., Yıldırım, H., Aslan, S., Ekşi, G., et al. (2018). Illustrated flora of Turkey. Istanbul, Turkey: ANG Foundation Nezahat Gökyiğit Botanical Garden Publications, Vol. 2 (in Turkish).

Güner, A., Özhatay, N., Ekim, T., and Başer, K. H. C. (2000). Flora of Turkey and the east Aegean Islands (supplement II). Edinburg, Scotland: Edinburg University Press.

Güneș, S., Savran, A., Paksoy, M. Y., Koşar, M., and Çakılcıoğlu, U. (2017). Ethnopharmacological survey of medicinal plants in Karaisalı and its surrounding (Adana-Turkey). J. Herb. Med. 8, 68-75. doi:10.1016/j.hermed.2017.04.002 
Gürbüz, İ., Gençler Özkan, A. M., Akaydin, G., Salihoğlu, E., Günbatan, T., Demirci, F., et al. (2019). Folk medicine in düzce province (Turkey). Turk. J. Bot. 43, 769-784. doi:10.3906/bot-1905-13

Hamilton, A. C. (2004). Medicinal plants, conservation and livelihoods. Biodivers. Conserv. 13, 1477-1517. doi:10.1023/B:BIOC.0000021333.23413.42

Heinrich, M. (2000). Ethnobotany and its role in drug development. Phytother Res. 14 (7), 479-488. doi:10.1002/1099-1573(200011)14:7<479::aid-ptr958>3.0.co;2-2

International Society of Ethnobiology (ISE) (2008). International society of ethnobiology code of ethics. Available at: https://www.ethnobiology.net/what-wedo/core-programs/ise-ethics-program/code-of-ethics/ (Accessed September 28, 2020).

İzol, E., Temel, H., Yilmaz, M. A., Yener, I., Olmez, O. T., Kaplaner, E., et al. (2020). A detailed chemical and biological investigation of twelve Allium species from Eastern Anatolia with chemometric studies. Chem. Biodivers. 18, e2000560. doi: $10.1002 / \mathrm{cbdv} .202000560$

Jaccard, P. (1908). Nouvelles recherches sur la distribution florale. Bull. Soc. Vaudoise Sci. Nat. 44, 223-270.

Karakaya, S., Polat, A., Aksakal, Ö., Sümbüllü, Y. Z., and Incekara, Ü. (2019). An ethnobotanical investigation on medicinal plants in South of Erzurum (Turkey). Ethnobot. Res. Appl. 18, 13. doi:10.32859/era.18.13.1-18

Karc1, E., Gürbüz, İ., Akaydın, G., and Günbatan, T. (2017). Folk medicines of Bafra (Samsun-Turkey). Turk. J. Biochem. 42 (4), 381-399. doi:10.1515/tjb-2017-0172

Kartal, Ç., and Güneş, F. (2017). Medicinal plants used in meriç town from Turkey. Ijper 51 (3), s249-s253. doi:10.5530/ijper.51.3s.23

Kaval, I., Behçet, L., and Cakilcioglu, U. (2014). Ethnobotanical study on medicinal plants in Geçitli and its surrounding (Hakkari-Turkey). J. Ethnopharmacol. 155, 171-184. doi:10.1016/j.jep.2014.05.014

Kaya, Ö. F., Dağlı, M., and Çelik, H. T. (2020). An ethnobotanical research in Şanlıurfa central district and attached Villages (Turkey). Indian J. Tradit. Knowl. 19 (1), 7-23.

Kheder, F. B. H., Mahjoub, M. A., Zaghrouni, F., Kwaja, S., Helal, A. N., and Mighri, Z. (2014). Chemical composition antioxidant and antimicrobial activities of the essential oils of Matricaria aurea Loef l. Growing in Tunisia. J. Essential Oil Bear. Plants 17 (3), 493-505. doi:10.1080/0972060X.2014.884777

Korkmaz, M., Karakuş, S., Selvi, S., and Çakılcıoğlu, U. (2016). Traditional knowledge on wild plants in Üzümlü (Erzincan-Turkey). Indian J. Tradit. Knowl. 15 (4), 538-545.

Kılıç, M., Yıldız, K., and Kılıç, F. M. (2020). Traditional uses of medicinal plants in Artuklu, Turkey. Hum. Ecol. 48, 619-632. doi:10.1007/s10745-020-00180-2

Miton, H., Claidière, N., and Mercier, H. (2015). Universal cognitive mechanisms explain the cultural success of bloodletting. Evol. Hum. Behav. 36 (4), 303-312. doi:10.1016/j.evolhumbehav.2015.01.003

Miyake, Y., Maeda, O., Tanno, K., Hongo, H., and Gündem, C. Y. (2012). New excavations at Hasankeyf Höyük: a 10th millennium cal. BC site on the upper Tigris, Southeast Anatolia. Neo-Lithics 1 (12), 3-7.

Mükemre, M., Behçet, L., and Çakılcıoğlu, U. (2015). Ethnobotanical study on medicinal plants in villages of Çatak (Van-Turkey). J. Ethnopharmacol. 166, 361-374. doi:10.1016/j.jep.2015.03.040

Nacakçı, F. M., and Dutkuner, İ. (2018). A study of ethnobotany in Kumluca (Antalya). Turk. J. For. 19 (2), 113-119. doi:10.18182/tjf.421970

Nadiroğlu, M., Behçet, L., and Çakılcıŏlu, U. (2019). An ethnobotanical survey of medicinal plants in Karlova (Bingöl-Turkey). Indian J. Tradit. Knowl. 18 (1), 76-87.

Özdemir, E., and Kültür, Ş. (2017). Wild edible plants of savaştepe district (Ballkesir, Turkey). Marmara Pharm. J. 21 (3), 578. doi:10.12991/marupj.319328

Özgen, N. (2011). "Preservation of the historical town of Hasankeyf within the scope of sustainable tourism," in Natural environment and culture in the mediterranian region II. Editors R. Efe, M. Öztürk, and İ. Atalay (Cambridge, United Kingdom: Cambridge Scholars Publishing).

Paksoy, M. Y., Selvi, S., and Savran, A. (2016). Ethnopharmacological survey of medicinal plants in Ulukışla (Niğde-Turkey). J. Herb. Med. 6 (1), 42-48. doi:10. 1016/j.hermed.2015.04.003

Panomket, P., Wanram, S., Srivorasmas, T., and Pongprom, N. (2012). Bioactivity of plant extracts against Burkholderia pseudomallei. Asian Biomed. 6 (4), 619-623. doi:10.5372/1905-7415.0604.100

Phillips, O., Gentry, A. H., Reynel, C., Wilkin, P., and Galvez-Durand, B. C. (1994). Quantitative ethnobotany and Amazonian conservation. Conserv. Biol. 8, 225-248. doi:10.1046/j.1523-1739.1994.08010225.x
Pieroni, A., Ahmed, H. M., and Zahir, H. (2017). The spring has arrived: traditional wild vegetables gathered by Yarsanis (Ahl-e Haqq) and Sunni Muslims in Western Hawraman, SE Kurdistan (Iraq). Acta Soc. Bot. Pol. 86, 3519. doi:10. 5586/asbp. 3519

Pieroni, A., Sõukand, R., Amin, H. I. M., Zahir, H., and Kukk, T. (2018). Celebrating multi-religious co-existence in Central Kurdistan: the bioculturally diverse traditional gathering of wild vegetables among Yazidis, Assyrians, and Muslim Kurds. Hum. Ecol. 46, 217-227. doi:10.1007/s10745018-9978-x

Pinar, S. M., and Behçet, L. (2014). Onopordum hasankeyfense (Asteraceae), a new species from South-eastern Turkey. Turk. J. Bot. 38, 226-233. doi:10.3906/bot$1303-56$

Polat, R. (2019). Ethnobotanical study on medicinal plants in Bingöl (City center) (Turkey). J. Herb. Med. 16, 100211-101121. doi:10.1016/j.hermed.2018.01.007

Polat, R., Cakilcioglu, U., and Satıl, F. (2013). Traditional uses of medicinal plants in Solhan (Bingöl-Turkey). J. Ethnopharmacol. 148, 951-963. doi:10.1016/j.jep. 2013.05.050

Sargin, S. A., and Büyükcengiz, M. (2019). Plants used in ethnomedicinal practices in Gulnar district of Mersin, Turkey. J. Herb. Med. 15, 100224. doi:10.1016/j. hermed.2018.06.003

The Plant List (2013). Available at: http://www.theplantlist.org (Accessed September 8, 2020).

Trotter, R. T., and Logan, M. H. (1986). "Informant census: a new approach for identifying potentially effective medicinal plants," in Plants in indigenous medicine and diet. Editor N. L. Etkin (Bedford Hill, NY: Redgrave), 91-112.

Tuzlac1, E. (2016). Traditional medicine Guide of Plants of Turkey. Istanbul, Turkey: Medical Bookstore publication.(in Turkish)

Tuzlaci, E., and Doğan, A. (2010). Turkish folk medicinal plants,IX: ovacik (Tunceli). Mpj 3, 136-143. doi:10.12991/201014449

Uzun, M., and Kaya, A. (2016). Ethnobotanical research of medicinal plants in Mihalgazi (Eskișehir, Turkey). Pharm. Biol. 54 (12), 2922-2932. doi:10.1080/ 13880209.2016.1194863

Vandebroek, I., Pieroni, A., Stepp, J. R., Hanazaki, N., Ladio, A., Alves, R. R. N., et al. (2020). Reshaping the future of ethnobiology research after the COVID-19 pandemic. Native Plants 6, 723-730. doi:10.1038/s41477020-0691-6

World Health Organization (WHO) (1993). Research guidelines for evaluating the safety and efficacy of herbal medicines. World Health Organization Regional Office for the Western Pacific, Manila. Available at: https://apps.who.int/iris/ handle/10665/207008 (Accessed January 30, 2021).

Yeşil, Y., and Akalın, E. (2009). Folk medicinal plants in Kürecik area (Akçadağ/ Malatya-Turkey). Turk. J. Pharm. Sci. 6, 207-220.

Yeşil, Y., and Akalın, E. (2010). The use of edible plants in Kürecik (Akçadağ/ Malatya). Istanbul. J. Pharm. 41, 90-103.

Yeşil, Y., Çelik, M., and Yılmaz, B. (2019). Wild edible plants in Yeşilli (MardinTurkey), a multicultural area. J. Ethnobiol. Ethnomed. 15, 52. doi:10.1186/ s13002-019-0327-y

Yeşil, Y., and İnal, İ. (2019). Traditional knowledge of wild edible plants in Hasankeyf (Batman Province, Turkey). Acta Soc. Bot. Pol. 88 (3), 3633. doi:10.5586/asbp. 3633

Yeşilada, E. (2002). "Biodiversity in Turkish folk medicine," in Biodiversity. Editor B. Şener (Boston, MA: Springer).

Yeșilada, E. (2005). Past and future contributions to traditional medicine in the health care system of the Middle-East. J. Ethnopharmacol. 100, 135-137. doi:10. 1016/j.jep.2005.06.003

Conflict of Interest: The authors declare that the research was conducted in the absence of any commercial or financial relationships that could be construed as a potential conflict of interest.

Copyright () 2021 Yeșil and İnal. This is an open-access article distributed under the terms of the Creative Commons Attribution License (CC BY). The use, distribution or reproduction in other forums is permitted, provided the original author $(s)$ and the copyright owner(s) are credited and that the original publication in this journal is cited, in accordance with accepted academic practice. No use, distribution or reproduction is permitted which does not comply with these terms. 


\section{APPENDIX A}

(1) Name and surname of the participant

(2) Age and sex of the participant

(3) Telephone and address of the participant

(4) Educational level of the participant

(5) Date of interview

(6) Place of residence of the participant

(7) Duration of residence of the participant
(8) What is the local name of the plant used?

(9) For which diseases do you use the plant?

(10) Which parts of the plant do you use (root, stem, flower, leaves, fruit, etc.)?

(11) How do you prepare the plant for use?

(12) How and when do you use the plant?

(13) Which group of age can use the plant?

(14) Approximately what dose do you use?

(15) How long does the convalescence period take?

(16) Did any complication occur from the plants you used? 